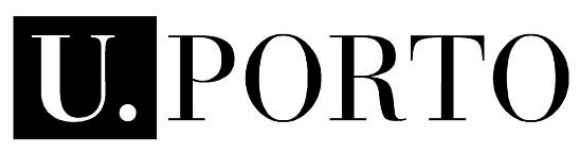

FC faculdade de ciências

\title{
Rheumatic Fever
}

Characterization

Based on indi-

cators extracted

from Echocar-

diograms exams

\section{Luiz Paulo M. Pires}

Computer Science Masters Data Mining Specialization

Faculty of Science, University of Porto

2018

\section{Supervisor}

Miguel Tavares Coimbra
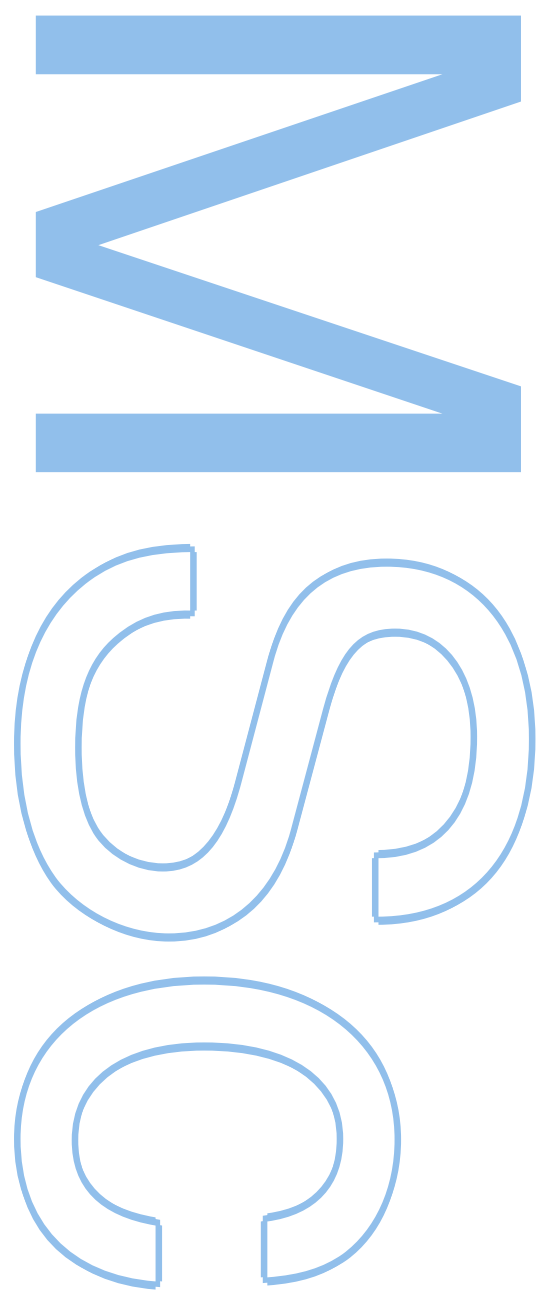


\section{U.PORTO

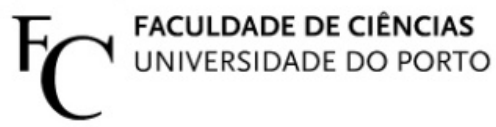

Todas as correções determinadas pelo júri, e só essas, foram efetuadas. O Presidente do Júri,

Porto,
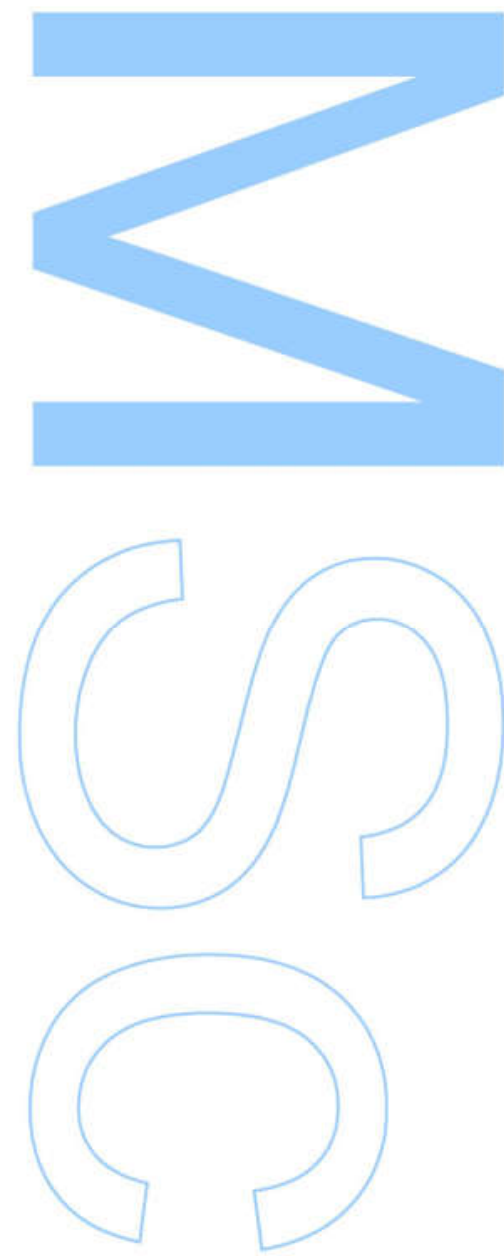


\section{Acknowledgements}

I would like to thank my supervisor Prof. Miguel Coimbra for accepting to supervise my thesis, providing time and expertise and pushing me to new territories. It was a challenging experience, but very rewarding. I would like to thank Malik Sultan for his technical advice, general guidance and friendship during this entire process.

None of this would be possible without my wife. Her support, patience through every step was instrumental to allow me to get to this point. She was also a great reviewer giving me nice pointers and suggestions. I'm also thankful for my family for providing me with moral support for my Master studies and my life in general.

I would also like to thank the Instituto de Telecomunicações - IT for providing me with the dataset used in this thesis, allowing me to do the necessary research with it. 


\begin{abstract}
Rheumatic heart disease is the serious consequence of repeated episodes of acute rheumatic fever. It is the major cause of heart valve damage resulting in morbidity and mortality. Its early detection is considered vital to control the disease's progression. The key manifestations that are visible in the early stages of this disease are changes in the thickness, shape and mobility of the mitral valve leaflets. Echocardiography based screening is sensitive enough to identify these changes in early stages of the disease.

In this thesis, an automatic approach is proposed to measure, quantify and analyse the thickness of the anterior mitral leaflet, in an echocardiographic video. The shape of the anterior mitral leaflet is simplified via morphological skeletonization and spline modelling to get the central line of the leaflet. To analyse the overall thickness from the tip to its base, the anterior mitral leaflet is divided into four quartiles. In each quartile the thickness is measured as the length of the line segment resulting from the intersection of the contour with the normal direction of the central point of each quartile. Finally, the thickness is analysed by measuring the variance per quartile, divided by leaflet position (open, straight and closed). The comparison between the normal and pathological leaflets are also presented, exhibiting statistical significant differences in all quartiles, especially near the tip of the leaflet.

An automatic approach for pathology classification based on motion patterns anterior mitral leaflet, as observed in an echocardiographic video, is also proposed. The motion points are extracted from the morphological skeleton of the anterior mitral leaflet and motion velocity and directionality is observed. This data is processed with the Discrete Fourier Transform, and used by several types of classifiers to perform the pathology classification. Finally the classifiers results are analysed and compared, presenting the best classifier results and motion features that were most distinguishable.
\end{abstract}




\section{Contents}

1 Introduction 1

1.1 Motivation . . . . . . . . . . . . . . . . . . . . . . . 1

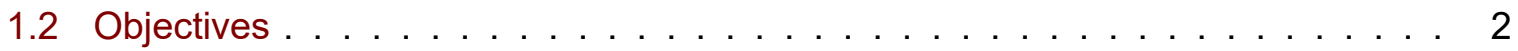

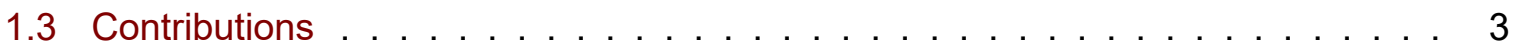

1.4 Thesis Structure $\ldots \ldots \ldots \ldots \ldots \ldots$

2 Screening Rheumatic Heart Disease $\quad 4$

2.1 Echocardiogram . . . . . . . . . . . . . . . . . . . . . 4

2.2 Screening Protocols . . . . . . . . . . . . . . . . . . 5

2.3 Discussion . . . . . . . . . . . . . . . . . . . 9

3 State-of-the-Art 10

3.1 Introduction . . . . . . . . . . . . . . . . . . . 10

3.2 Thickness analysis of the AML . . . . . . . . . . . . . 10

3.3 Motion analysis of the $\mathrm{AML} \ldots \ldots \ldots \ldots \ldots \ldots$

3.4 Discussion . . . . . . . . . . . . . . . . . . . . . . . 15

4 Thickness Profile Extraction $\quad 17$

4.1 Introduction . . . . . . . . . . . . . . . . . . 17

4.2 Materials . . . . . . . . . . . . . . . . . . . 17

4.3 Framework . . . . . . . . . . . . . . . . . . . . . 18

4.3 .1 Skeletonization . . . . . . . . . . . . . . . . . . . . 19

4.3 .2 Spline Modelling . . . . . . . . . . . . . . . . . . . . . . . 21

4.3.3 Thickness Measurements . . . . . . . . . . . . . . . . . . . . . 23

4.3 .4 Position Detection . . . . . . . . . . . . . . . . . . . . . 23

4.4 Results . . . . . . . . . . . . . . . . . . . . . . . . . . . . . 24

4.4.1 Average Thickness by Quartile . . . . . . . . . . . . . . . . . . . 25

4.4.2 Normal Versus Pathological Thickness Profiles . . . . . . . . . . . . . . . 25

4.4 .3 Quartile Versus Position . . . . . . . . . . . . . . . . . . . . . . . . . 27

4.5 Discussion . . . . . . . . . . . . . . . . . . 28 
5 Motion Classification $\quad 29$

5.1 Introduction . . . . . . . . . . . . . . . . . . . . . . . 29

5.2 Materials . . . . . . . . . . . . . . . . . . . . . . . 29

5.3 Framework . . . . . . . . . . . . . . . . . 30

5.3 .1 Skeletonization . . . . . . . . . . . . . . . . . . 30

5.3 .2 Initial Feature Extraction . . . . . . . . . . . . . . . . . . . . . . . 31

5.3 .3 Frame Rate Normalization . . . . . . . . . . . . . . . . . . . 32

5.3 .4 Discrete Fourier Transform (DFT) . . . . . . . . . . . . . . . . 33

5.3 .5 Classification . . . . . . . . . . . . . . . . 35

5.4 Results . . . . . . . . . . . . . . . . . . . . 42

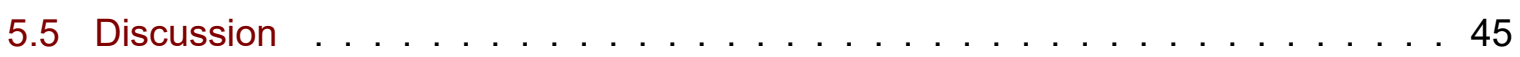

6 Conclusion $\quad 46$

6.1 Discussion . . . . . . . . . . . . . . . . . . . 46

6.2 Future Work . . . . . . . . . . . . . . . . . . . . . . . 48

$\begin{array}{ll}\text { Bibliography } & 49\end{array}$ 


\section{List of Tables}

4.1 Median Thickness . . . . . . . . . . . . . . . . . . . . . . . 26

4.2 Thickness Variance Between Pathologies . . . . . . . . . . . . . . . . . 28

5.1 Evaluation Metrics by Features . . . . . . . . . . . . . . . . . . . . . . 42

5.2 Combined Features Results . . . . . . . . . . . . . . . . . . . . . . . 44 


\section{List of Figures}

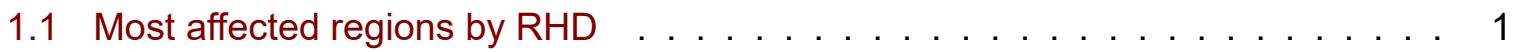

2.1 Most common Echocardiograms . . . . . . . . . . . . . . . . . . . . 6

2.2 M-Mode Source . . . . . . . . . . . . . . . . . . . . 7

2.3 Doppler Color Flow . . . . . . . . . . . . . . . . . . . . . . . . . . . . . 7

2.4 Three-dimensional Ultrasound images . . . . . . . . . . . . . . . . . . . . . 8

3.1 N. Subramanian's approach for septum segmentation . . . . . . . . . . . . . . 11

3.2 N. Subramanian's Septum Thickness Measurement . . . . . . . . . . . . . . . 11

3.3 Dhutia's Doppler beats measurement . . . . . . . . . . . . . . . . . . . . 13

3.4 Chen's proposed framework . . . . . . . . . . . . . . . . . . . 15

4.1 The Thickness Profile Extraction Framework . . . . . . . . . . . . . . . . 18

4.2 Skeletonization . . . . . . . . . . . . . . . . . . . . . . 19

4.3 Zhang-Suen Neighbor Pixels Mapping . . . . . . . . . . . . . . . . . . . . 19

4.4 Zhang-Suen 01 Pattern Example . . . . . . . . . . . . . . . . . . . . . . . . 20

4.5 K3M Simplified Flowchart . . . . . . . . . . . . . . . . . . . . . . . . 22

4.6 Spline Modelling . . . . . . . . . . . . . . . . . . . . . . . . . . . 24

4.7 Thickness Measurements . . . . . . . . . . . . . . . . . . . . 25

4.8 AML Thickness Profile . . . . . . . . . . . . . . . . . . . . . . 26

4.9 AML Thickness Profile Normal x Pathological . . . . . . . . . . . . . . . . . 27

5.1 The Thickness Profile Extraction Framework . . . . . . . . . . . . . . . . . 30

5.2 Extracted Motion Features . . . . . . . . . . . . . . . . . . . . . . . . . 34

5.3 DFT of Motion Features . . . . . . . . . . . . . . . . . . . . 36

5.4 C5.0 Resulting Decision Tree . . . . . . . . . . . . . . . . . . . . 38

5.5 Support Vector Machine $(\mathrm{SVM}) \ldots \ldots$. . . . . . . . . . . . . . . 38

5.6 Ensemble's Fundamental Reasons . . . . . . . . . . . . . . . . . . . . . . . . . 39

5.7 Dynamic Time Warping (DTW) . . . . . . . . . . . . . . . . . . . . 41

5.8 Hausdorff's Distance . . . . . . . . . . . . . . . . . . . . . . . . . . . . 41

5.9 Evaluation Metrics by Feature - Graphical Comparison . . . . . . . . . . . . . . . 43

5.10 Combined Features Results - Graphical Comparison . . . . . . . . . . . . . . . . 44 
6.1 Manual x Automatic Segmentation Comparison . . . . . . . . . . . . . . . 47 


\section{Abbreviations}

$\begin{array}{ll}\text { AML } & \text { Anterior Mitral Leaflet. } \\ \text { ARF } & \text { Acute Rheumatic Fever. } \\ \text { ASE } & \text { American Society of Echocardiography. } \\ \text { DFT } & \text { Discrete Fourier Transform. } \\ \text { DTW } & \text { Dynamic Time Warping. } \\ \text { DWT } & \text { Discrete Wavelet Transform. } \\ \text { FFT } & \text { Fast Fourier Transform. } \\ \text { FPR } & \text { False Positive Rate. } \\ \text { HAND } & \text { Handheld Echocardiography. } \\ \text { k-NN } & \text { k-Nearest Neighbor. } \\ \text { LCSCA } & \text { Least-squares Cubic Spline Curves Approxi- } \\ & \text { mation. } \\ \text { PCA } & \text { Principal Components Analysis. } \\ \text { PLAX } & \text { Parasternal long-axis. } \\ \text { RHD } & \text { Rheumatic Heart Disease. } \\ \text { SMID } & \text { Single Instruction Multiple Data. } \\ \text { SMOTE } & \text { Synthetic Minority Over-Sampling Technique. } \\ \text { SRC } & \text { Sparse Representation Classification. } \\ \text { SSE } & \text { Squared Standard Error. } \\ \text { STAND } & \text { Standard Portable Echocardiography. } \\ \text { SVM } & \text { Support Vector Machine. } \\ \text { TPR } & \text { True Positive Rate. } \\ \text { WHF } & \text { World Heart Federation. } \\ \text { WHO } & \text { World Health Organization. }\end{array}$




\section{Chapter 1}

\section{Introduction}

\subsection{Motivation}

Rheumatic Heart Disease (RHD) is the serious consequence of repeated episodes of Acute Rheumatic Fever (ARF), an autoimmune reaction of a group A streptococcal infection [1]. It is estimated that $60 \%$ of all ARF cases will develop RHD. RHD is the major cause of heart valve damage and causes over 200.000 deaths per year [2].

This disease starts manifesting at a young age, and it is estimated that over 400.000 annual cases in children (ages 5-14) in less developed countries, while little over 22.000 annual cases in more developed countries [2]. These numbers show how this disease's burden is mostly shouldered by less developed countries.

Early detection enables prevention of the progression of the disease using penicillin based prophylactics injections [3], for a period of up to 10 years [2].

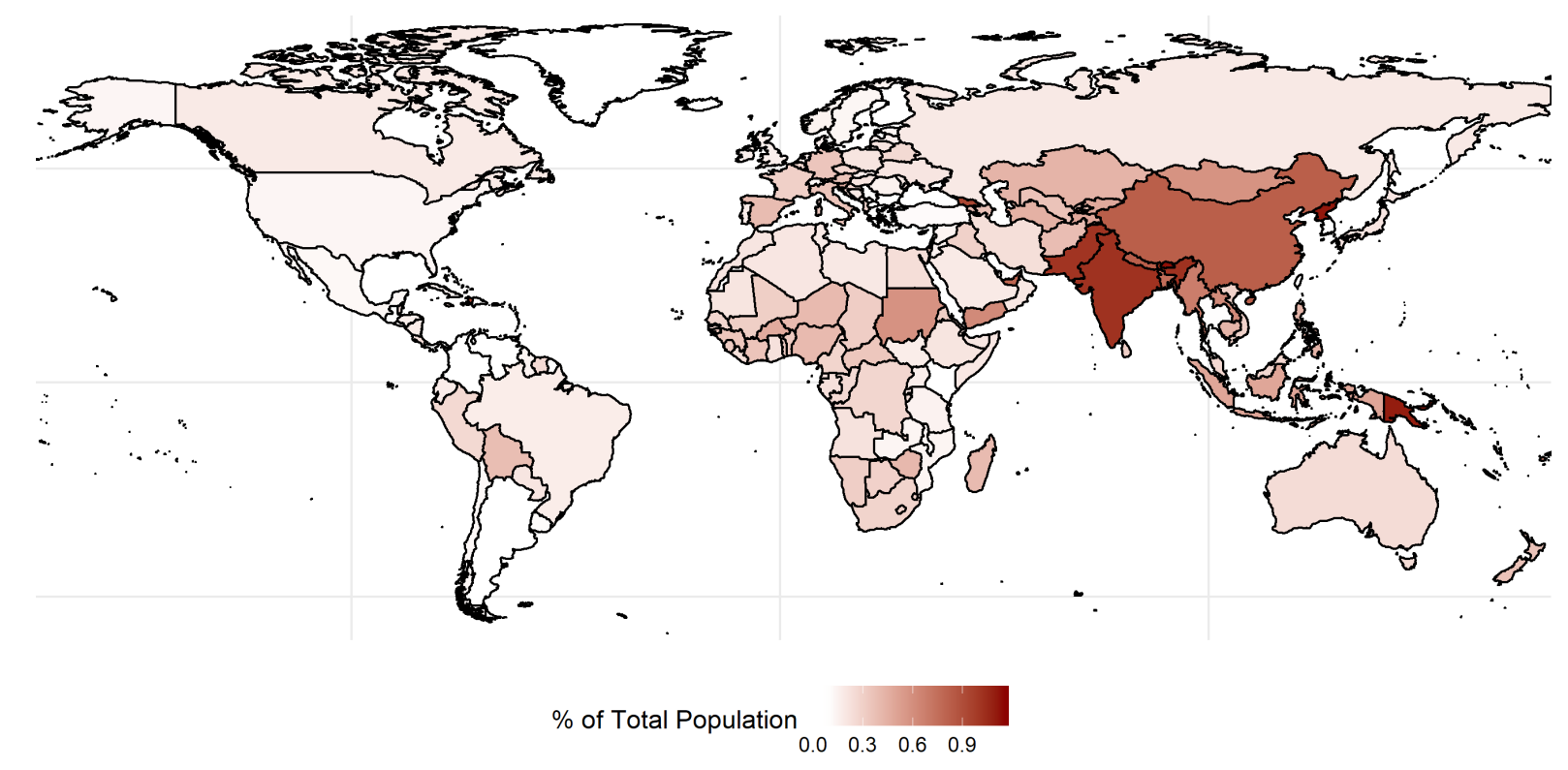

Figure 1.1: Most affected regions by RHD - Source: World Health Organization (WHO) 
Technological advances made available echocardiography, providing an alternative to the stethoscope, while requiring less experience by the physician to perform the screening and being more sensitive [4] and also being noninvasive [3]. Further advancements reduced the costs of this technology and increased its portability, making it more suitable for less developed countries, with lower financial means.

Unfortunately the decrease in cases on more developed countries has led to a reduction in the research effort for this disease [5]. The current prophylactic treatment is based on findings of studies made in the United States of America in the 1950s. This shows the lack of specific medical research on this disease's treatment.

Although medical research on the treatment of RHD has dwindled, advancements on the fields of computer vision and machine learning have been progressing and providing help with the diagnosis, even as echocardiogram image analysis proves to be a complex challenge, due to the high amount of speckle noise and missing information between frames, making it particularly challenging to segment and track features [6].

The current guidelines defined by the World Heart Federation (WHF) aim to define the minimum echocardiographic criteria to perform the RHD diagnosis [3]. They provide metrics for pathological aortic and mitral regurgitation, mitral leaflet thickness chordal thickness and leaflet motion. As new literature is made available, providing data that can challenge these guidelines [7], suggest new criteria [8] or indicate limitations [9], a deeper analysis of these guidelines should be performed in order to improve it and in doing so, help physicians perform the RHD diagnosis with greater accuracy.

The mitral valve is one of the heart's four valves and is composed of the Anterior Mitral Leaflet $(A M L)$ and the Posterior Mitral Leaflet (PML). They are attached to the anterolateral and posteromedial muscles of the left ventricle, by small tendons know as chordae tendineae. As measurement of the PML has a lower interobserver agreement [3], segmentation and measurement efforts focus on the AML.

\subsection{Objectives}

The main objective of this Master Thesis is to research and develop novel computer vision algorithms that can extract relevant clinical information from an AML's shape and motion patterns. Obtaining these algorithms requires understanding of the AML's behaviour and physiology done by using a previously provided dataset of echocardiograms, building statistical models of AML shape and motion, and adequate experimental validation of algorithmic performance.

By pursuing this technical objective, new data can be provided which can help diagnosticians and lower the technical and experience requirements that are currently necessary for medical professionals to perform screening of the patients. This can be potentially useful for less developed countries as they tend to suffer from a lack of qualified physicians, especially 
in remote locations.

\subsection{Contributions}

During this work, a set of contributions were proposed in order to achieve the defined objectives. A brief description of these follows:

- Defined a stable, reproducible thickness extraction algorithm for the AML. A novel approached was proposed using a combination of skeletonization and spline modelling to achieve this.

- Validated the current WHF guidelines for ARF assessment, as well as other articles inputs, using the proposed thickness extraction technique. New conclusions were also established.

- Created an algorithm that analyses the AML's motion patterns.

\subsection{Thesis Structure}

This thesis is organized as follow:

- Chapter 2 focuses on the current medical protocols and guidelines for screening RHD, as well as providing background information on echocardiophy, which is the primary tool currently used for screening RHD.

- Chapter 3 discusses the state of the art on automatic echocardiogram analysis, as well as motion analysis.

- Chapter 4 thoroughly explains the proposed automatic thickness profile extraction algorithm and discusses the statistical data it generates based on the provided echocardiogram dataset.

- Chapter 5 is dedicated to the proposed motion analysis algorithm, as well as the classification algorithms used and the overall archived results.

- Chapter 6 draws the overall conclusion of the thesis. 


\section{Chapter 2}

\section{Screening Rheumatic Heart Disease}

On this chapter the Echocardiogram is described in further detailed as the algorithms proposed in this thesis are based on a dataset of echocardiograms, requiring a deeper understanding of this technology. The current echocardiogram-based screening protocols are also described, providing the information as to what features to observe in order to detect a pathological case.

\subsection{Echocardiogram}

An echocardiogram is a medical imaging technique that is based on emitting high frequency sound waves $(>20.000 \mathrm{~Hz})$ and using the reflection of these waves to produce images of the heart. Higher frequencies improve resolution of these images at the cost of decreased tissue penetration.

The produced images can be $2 \mathrm{D}$ or $3 \mathrm{D}$. By using the Doppler Effect (more specifically the phase shift), information of direction and velocity of the blood flow can be calculated and demonstrated visually, usually by mean of colour hues.

Developments of the echocardiogram technology have increased its portability from the original Standard Portable Echocardiography (STAND) to Handheld Echocardiography (HAND) and the newest Ultra-Portable Machines, which are basically a handheld ultrasound scanner and a tablet, such as the Philips Lumify line of products ${ }^{1}$. This was also followed by a drastic reduction of costs, reducing the expense on the purchase of these devices. This size reduction can initially lead to a lower resolution, which might make then unsuitable for RHD screening, but as the technology evolves, resolution improves as well.

There are 28 two-dimensional views that can be extracted from the heart using the echocardiogram equipment, although the following six, as depicted on Figure 2.1, are the most commonly used :

1. Aortic Valve - Short Axis

\footnotetext{
${ }^{1}$ Philips Lumify - https://www.lumify.philips.com/web/
} 


\section{Apical Four Chamber}

3. Apical Two Chamber

4. Left Parasternal - Long Axis

5. Left Ventricle - Short Axis

6. Subcostal

These two-dimensional views are used to detect abnormalities in shape and / or structure of the heart, as they are formed by an ultrasound arc of a cross-section of the heart.

The M-Mode uses a different approach, by pulsating a narrow bean of ultrasound through a simple point in the heart, which produces an image like Figure 2.2, that provides temporal and spatial information used the for assessment of valve motion, ventricular function, wall thickness and other temporal or spatial information analysis.

The Doppler ultrasound uses the Doppler effect to measure direction and velocity [11] of the blood, by analysing the frequency or phase shift of the moving cells in its interaction with the ultrasound waves. Colours are usually used to represent the velocity measurements. Figure 2.3 demonstrates an example of a Colour Flow Doppler of a patient with severe tricuspid regurgitation.

Three-Dimensional echocardiogram is a more recent development of the ultrasound technology, made possible by advancements on computer and transducer technologies. Using fully sampled matrix-array transducers alongside with modern hardware and software enables the production of three-dimensional ultrasound images. There are, currently, two methods used to produce these images: real-time and electrocardiographically triggered [12]. Real-time suffers from poor temporal and spatial resolution, while electrocardiographically triggered images suffer from artefacts produced by irregular motion. Figure 2.4 demonstrates the produced three-dimensional images of three different planes.

While the echocardiogram is a non-invasive technology that provides physicians with important information about the patient's heart condition, it also has significant flaws such as image quality and speckle noise. These aspects can lead to misdiagnoses. Expertise and experience are fundamental to reduce these errors, as these images are extremely sensitive to the operator's skill, which must be able to obtain the right view for each specific patient.

\subsection{Screening Protocols}

RHD screening protocols have evolved in the last 10 years, being initially developed based on best available evidence, until the release of the WHF guidelines [3] in 2012, which were formulated based on the work of 21 investigators, from six different continents, to formulate evidence-based guidelines, enabling rapid identification of RHD patients who had no previous history of ARF. 

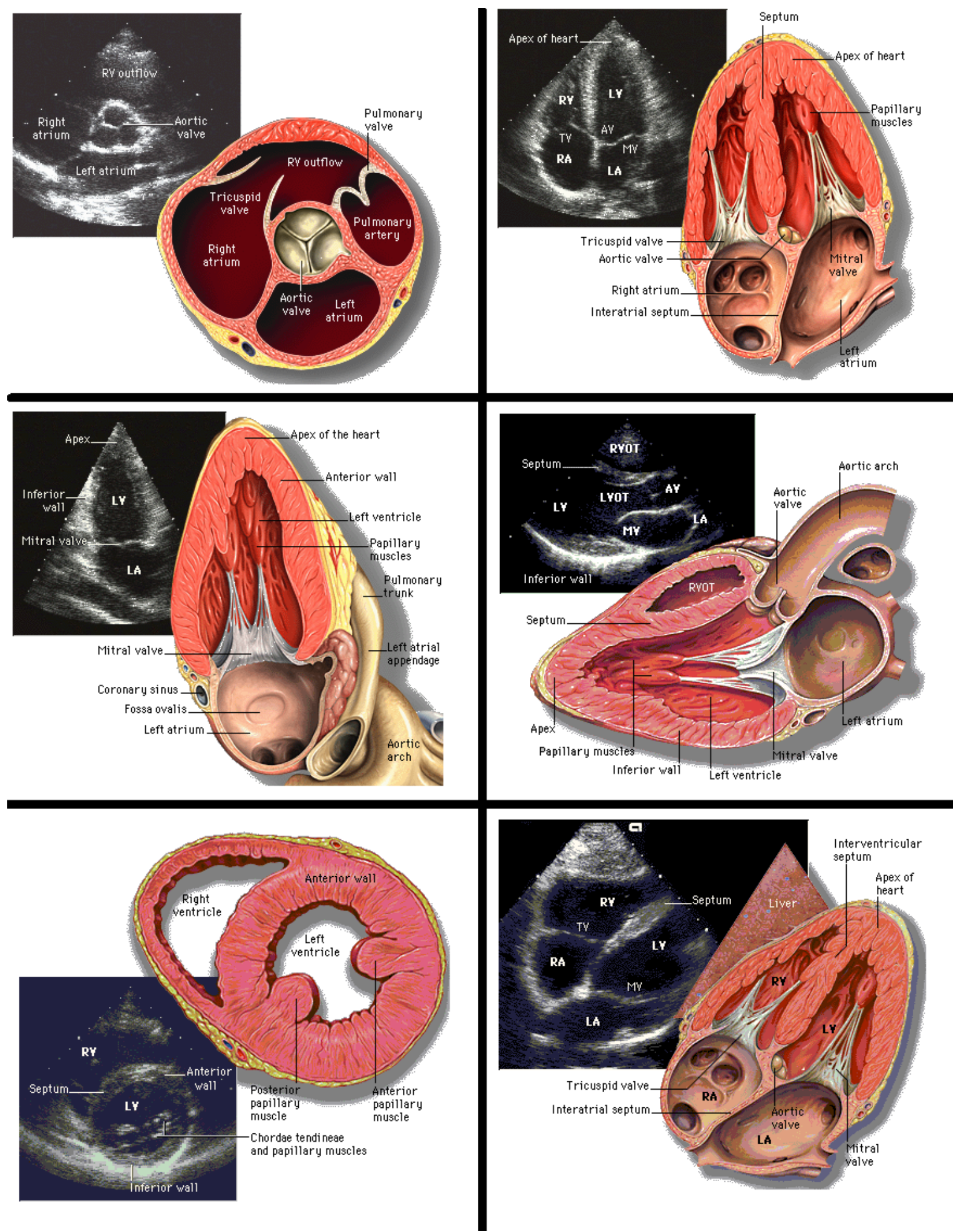

Figure 2.1: Most common Echocardiograms - (Adapted from Yale Echo Atlas) 


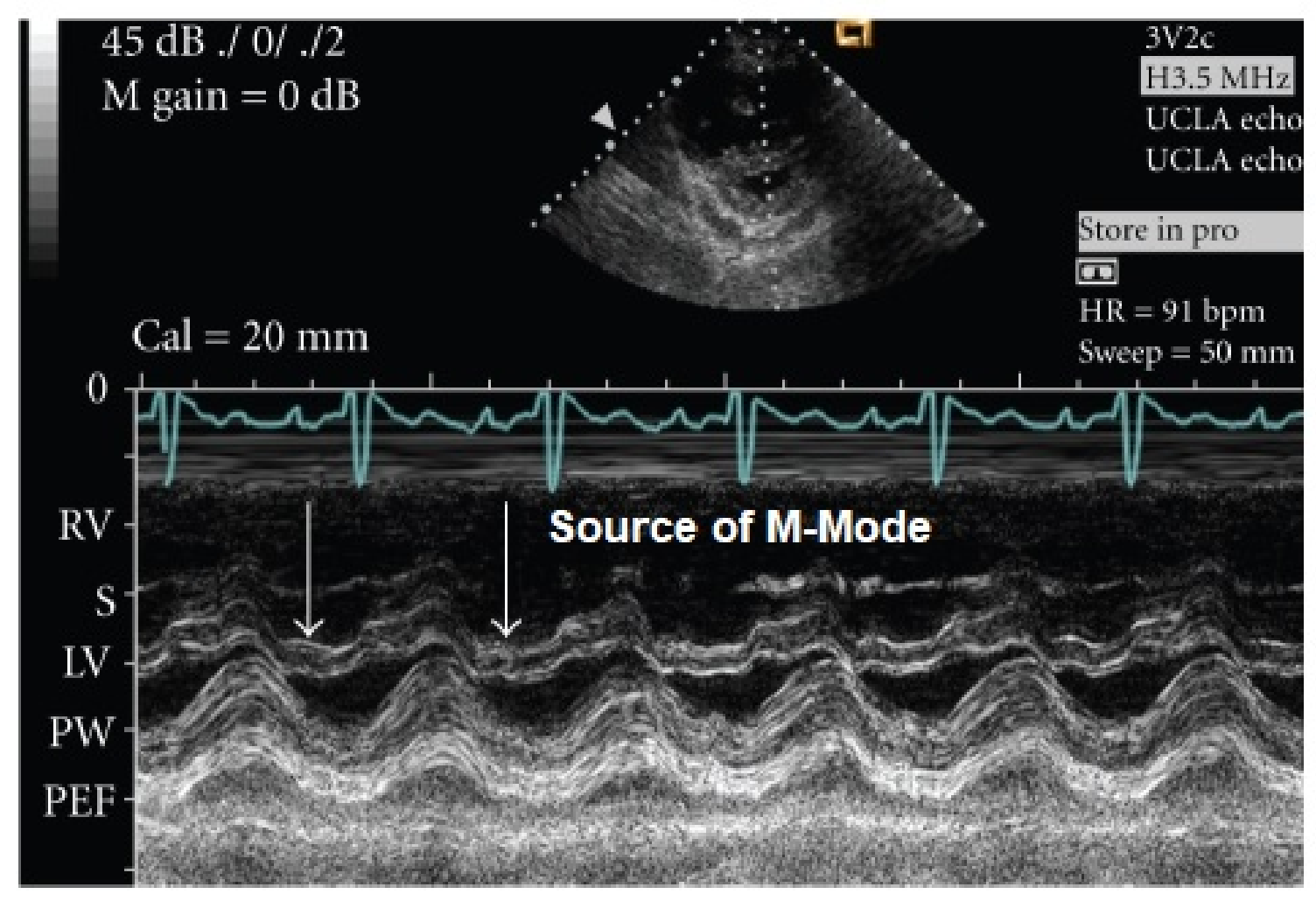

Figure 2.2: M-Mode Source (Adapted from [10])

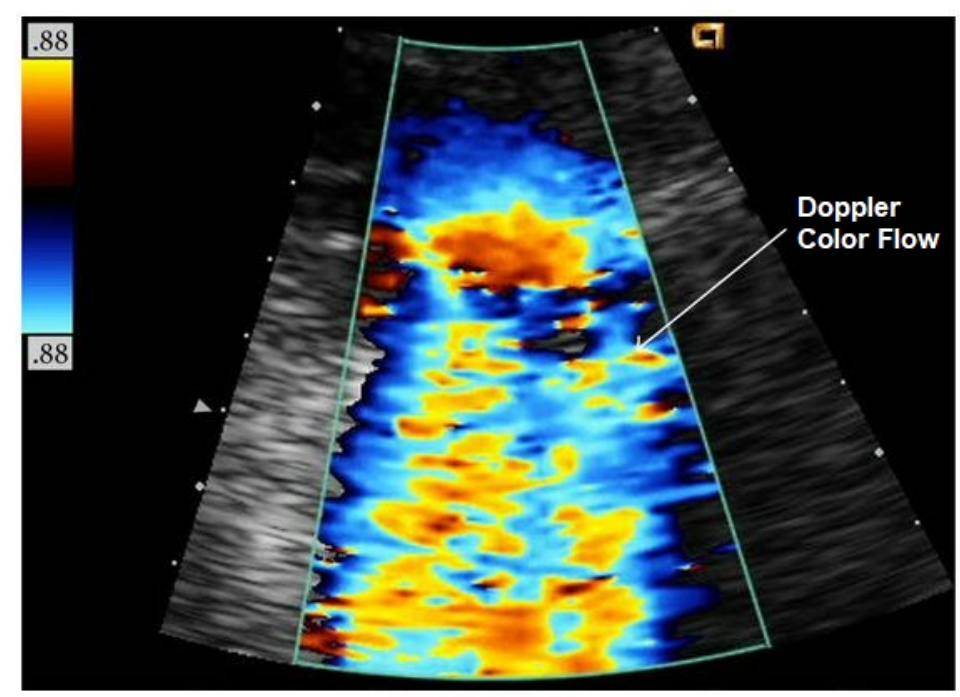

Figure 2.3: Doppler Color Flow (Adapted from [10])

These guidelines were important to define a minimum criteria for the analysis of echocardiograms, helping the physician to perform the diagnosis. The echocardiogram evidence should be analysed considering various factors, such as the patient's geographic location, ethnicity, 

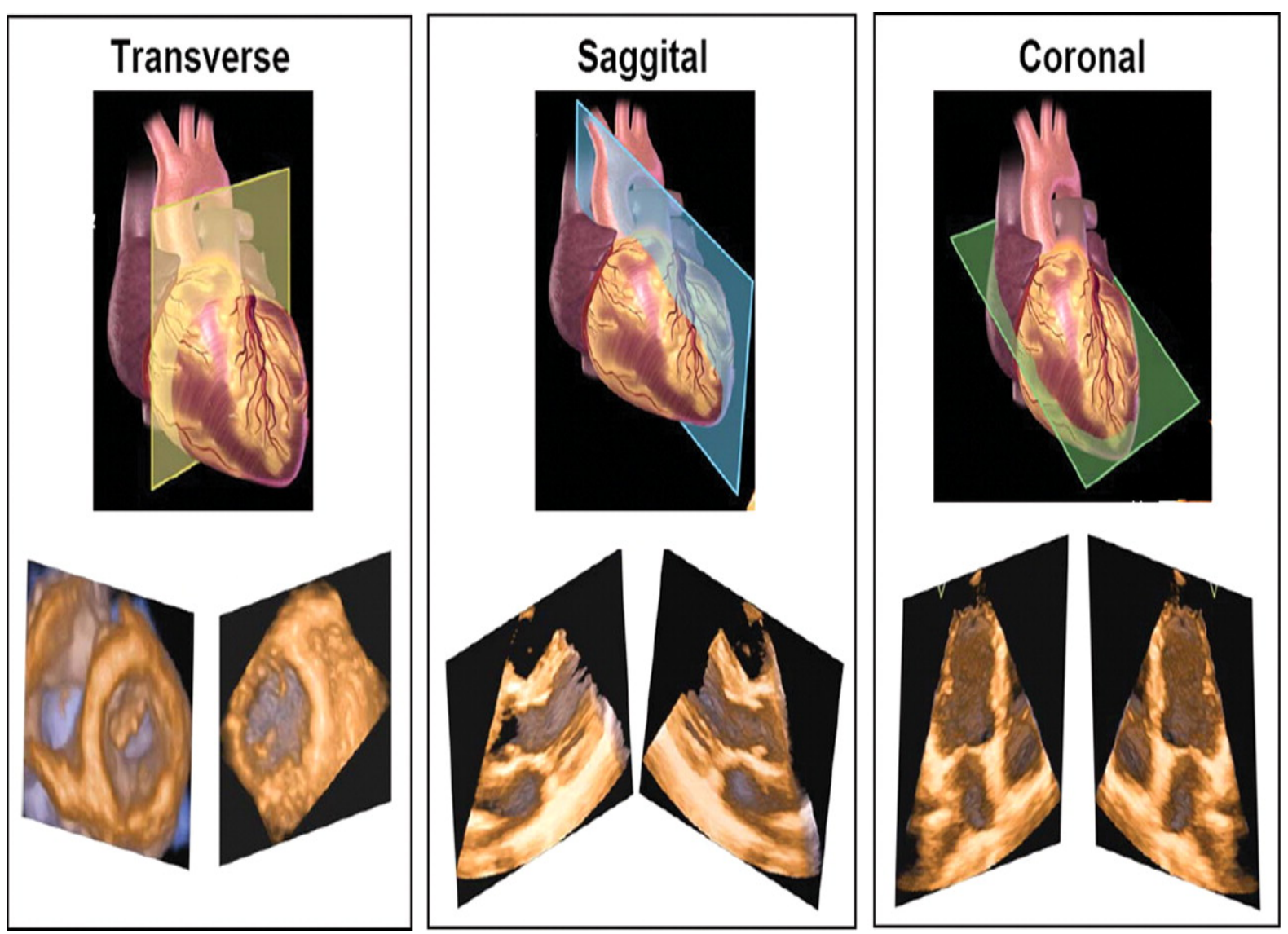

Figure 2.4: Three-dimensional Ultrasound images (Adapted from [12])

living condition, as well as other clinical factors.

The following items are considered most relevant, according to the WHF guidelines, when analysing echocardiograms:

- Mitral Valve Regurgitation

- Anterior Valve Regurgitation

- Mitral Valve Thickness

- Chordal Thickness

- Leaflet Motion

While the difference in the behaviour of these features in normal and pathological situations is well described in the WHF Guidelines [3], other articles indicate that some criteria could be improved to reduce inter and intra observer differences. One article in particular found that there were no significant statistical differences in the measurement of the leaflet whether at the tip or the midpoint [7], a result affected by a lack of clearly defined metrics or points of measurement of the leaflet, when analysing the leaflet's thickness. 
These measurement issues, image quality problems of echocardiograms, and external variables such as location, living conditions, etc, indicate the complexity of RHD diagnosis. The experience and expertise of the physician performing it are critical. But in less developed countries, with sparse resources, this proves to be another obstacle in reducing the burden of this disease.

The evolution of the electrocardiogram has also shown the need for verification and updates on these guidelines. With the appearance of HAND, new studies were performed to validate and adapt the WHF guidelines, which were established with STAND, to these news devices [9] [13], as they had a lower frame rate, lack of a continuous-wave Doppler and had $1 \mathrm{~mm}$ measurement increments, which made adaptations to the WHF Guidelines necessary. making then more suitable for screening and not a final diagnosis, at the described hardware generation.

The lower cost and increase portability of these HAND devices, allowed an increase in echocardiogram screenings on less developed countries, although the described hardware generation, HAND devices were more suitable for screening than to perform the final diagnosis. As new products are being developed, such as the mentioned ultra-portable devices, continuous validation and updates are required on the WHF guidelines, as well as further improvements to reduce the complexity and error of the RHD diagnosis.

\subsection{Discussion}

Echocardiogram is a powerful medical imaging technique, providing various views of the heart as well as other modes that provide information on the blood flow, wall thickness and various temporal and spatial data, enabling physicians to perform a thorough exam on the patient in a non-invasive manner and perform diagnosis for several types of heart conditions, including RHD. As the technology evolves it becomes an even more powerful tool.

As echocardiograms started to be more utilized for RHD diagnosis, different approaches on how to perform such diagnosis were published. In order to uniformize this, in 2012 the WHF developed a set of evidence-based guidelines, providing adequate guidance to physicians. However, since these guidelines were published, new technologies have been made available which can help improve the methods on how physicians can perform RHD diagnosis, as well as complement the WHF guidelines. 


\section{Chapter 3}

\section{State-of-the-Art}

\subsection{Introduction}

State of the art approaches to automatic measurement and motion analysis were searched on scholar databases such as PubMed, Web of Science and Scopus. Multiple queries were used with multiple terms such as "automatic thickness measurement", "automatic motion classification" and other similar terms.

While a direct reference for automatic measurement and analysis of the thickness of the AML was not found in the current literature, other automatic measurement approaches were analysed, providing hints and ideas to the work presented in this thesis.

\subsection{Thickness analysis of the AML}

N. Subramanian et. al. [14], in 2010, proposed a method for automatic interventricular septum thickness measurement, an important indicator of cardiac hypertrophy. Similar to other measurements on 2D echocardiogram images, it also suffers from a large inter and intra observer variability, which can lead to misdiagnosis. The initial segmentation of the septum was performed over a search space of two smooth 1D profiles, representing the top and bottom of the septum. The use of these two profiles enables the search for the 1D functions, while constraining the width of the septum and reducing computational costs. The segmentation process presumes a constant image intensity at the boundaries and uses Gaussian statistics to split the regions above and below the profiles, obtaining more accurate results as it adjusts the energy over the space of the 1D functions. Figure 3.1 provides the visual cues of this approach.

After the segmentation of the septum, a detection of the tip of the AML is performed by using a motion map over several frame, accounting for the large displacements that can occur due to missing data and identifying candidate locations. These candidate locations are then grouped using the K-means clustering algorithm, and the cluster centres are used as the AML's tip for the measurement procedure that follows. 

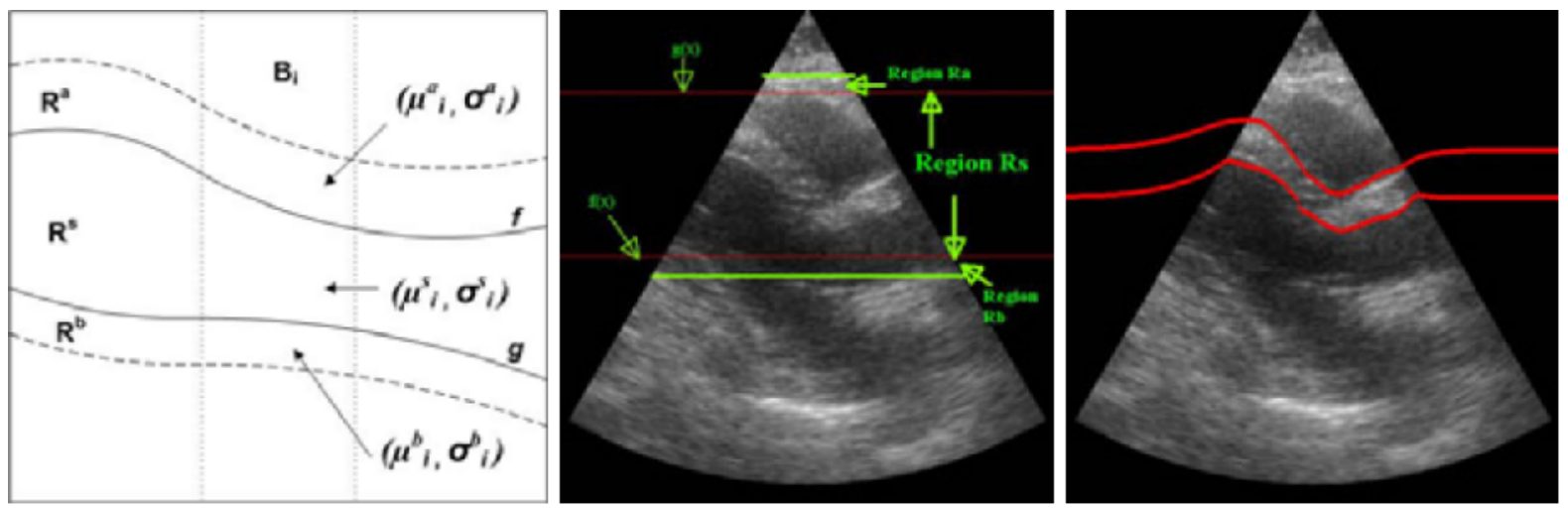

Figure 3.1: N. Subramanian's approach for septum segmentation (Adapted from [14])

The American Society of Echocardiography (ASE) manual regarding septum thickness measurement protocol specifies that the measurement should be made by drawing an orthogonal line between the centre of the septum through the AML's tip. To mimic this behaviour, the proposed algorithm calculates the medial axis by dividing the septum in X amounts of regions, where ten is the recommended value, and fitting lines through these segments by linear regression. Orthogonal lines between these line segments and the AML's tip are calculated. The line with the shortest Euclidean distance from the intersecting point of line segment and the AML's tip is used to measure the septum thickness, considering the distance between the point where the line intersects with the septum segmentation mask and the AML's tip. Figure 3.2 exhibits this method's results.

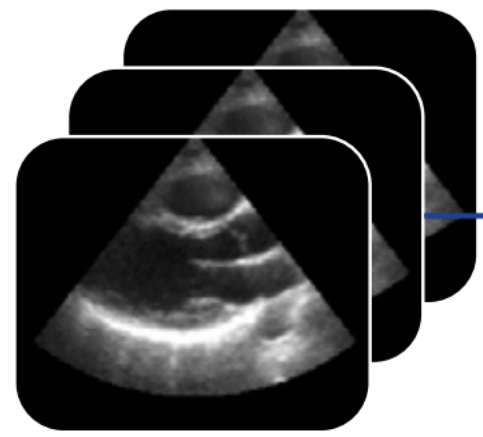

2D Echocardiogram

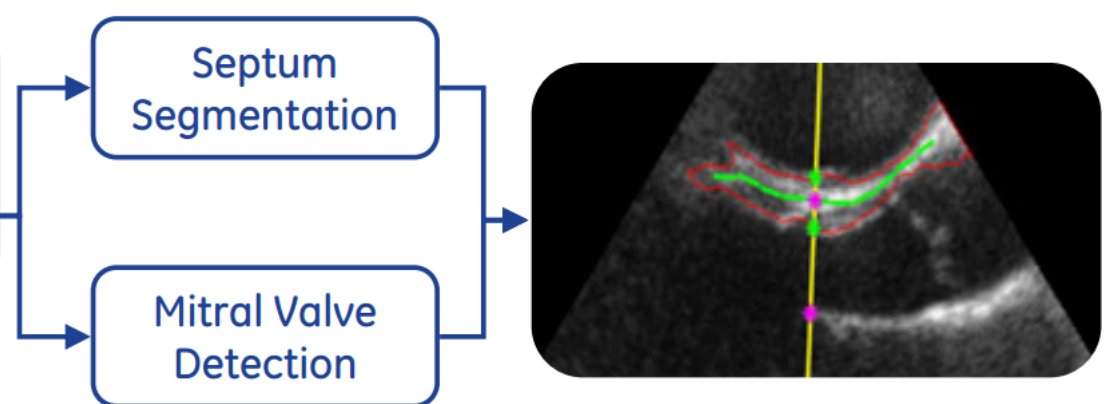

Septum Thickness Measurement

Figure 3.2: N. Subramanian's Septum Thickness Measurement (Adapted from [14])

This methodology used B-mode parasternal long axis recordings of 57 patients, with an average of 3 cardiac cycles per video. The automated results were compared to the manual measurements of two cardiologists. They run results in near real-time and were able to archive results of $89 \%$ and $82 \%$ match to the manual data, considering a $2 \mathrm{~mm}$ tolerance.

While these results are interesting, the dataset is poorly discussed, so the image quality of the overall dataset is unknown, and it uses algorithms that can yield vastly different results on different images such as the K-means used in the AML's tip detection. The K parameter 
is not discussed, and the medial axis calculation that uses $X$ segments, which depending on their shape and orientation create instability when trying to determine the resulting orthogonal line. The accepted tolerance for this case is far superior to the tolerance of the AML's thickness measurement, where $2 \mathrm{~mm}$ is already considered a thickened valve, making this method too imprecise for any practical usefulness.

Dhutia et. al. [15], in 2017, developed a method to perform automatic measurements on cardiac function by tissue Doppler, an echocardiograph technique that allows the physician to assess the left ventricular systolic function and the diastolic function. As manual measurements are time-consuming, tedious and error-prone, an automatic approach allows for the physician to focus on acquiring more Doppler beats, instead of spending time and energy measuring them. The proposed algorithm performs Otsu thresholding and a Butterworth low-pass filter on the acquired Doppler beats to improve the signal and remove noise. It then proceeds to obtain the highest intensity pixel of each column, entitled middle of the envelop, and the outer edge, with is the point with the sharpest drop in intensity, from the middle of the envelop to the outer region. Figure 3.3 illustrates this process.

The produced velocity curves were then smoothed with a Savitzky-Golay least-squares filter. Autocorrelation was then used to estimate the length of the cardiac cycles, information used to delimit the minimum distance between peaks, preventing from noise being classified as peak velocities. The resulting peaks were then classified as systolic and diastolic. The final measurement calculation was done from the middle of the envelop.

The results were based on a dataset composed of data acquired from 48 patients, 30 males, 18 females, with a mean age of 64 years, with 30 seconds of data recorded per patient, later compared to the manual annotation of three experts. The proposed algorithm was as similar to the experts, as they were from each other, but with the advantage of being highly reproducible since it doesn't suffer from intra observer variability, which represented a standard deviation of $0.71 \mathrm{~cm} / \mathrm{s}$ in this dataset, demonstrating how sensitive this data is to interpretation.

The proposed method used a well-defined dataset and performed precise statistical analysis. The threshold and filtering approach makes it computationally inexpensive, but less robust to stronger noise that can occur on ultrasound images. An approach using active contours like Sultan et. al. [16] would potentially be more robust.

\subsection{Motion analysis of the AML}

Motion analysis of the AML is a challenging subject duo to several factors: velocity of the AML's motion, speckle noise that deforms the AML's shape, large displacements and probe orientation, all of which can interfere with the overall result. Physicians try to overcome these limitations by obtaining more samples of the patient's AML motion by prolonging the examination and also by reviewing the recorded video.

Different approaches of motion analysis and classification were investigated. Ahmadi et. 


\section{Original tissue Doppler trace}

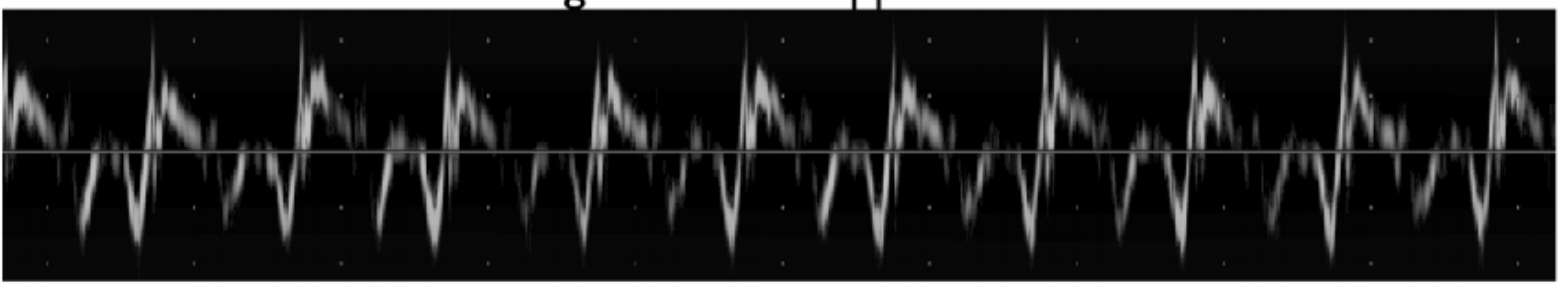

Thresholded trace
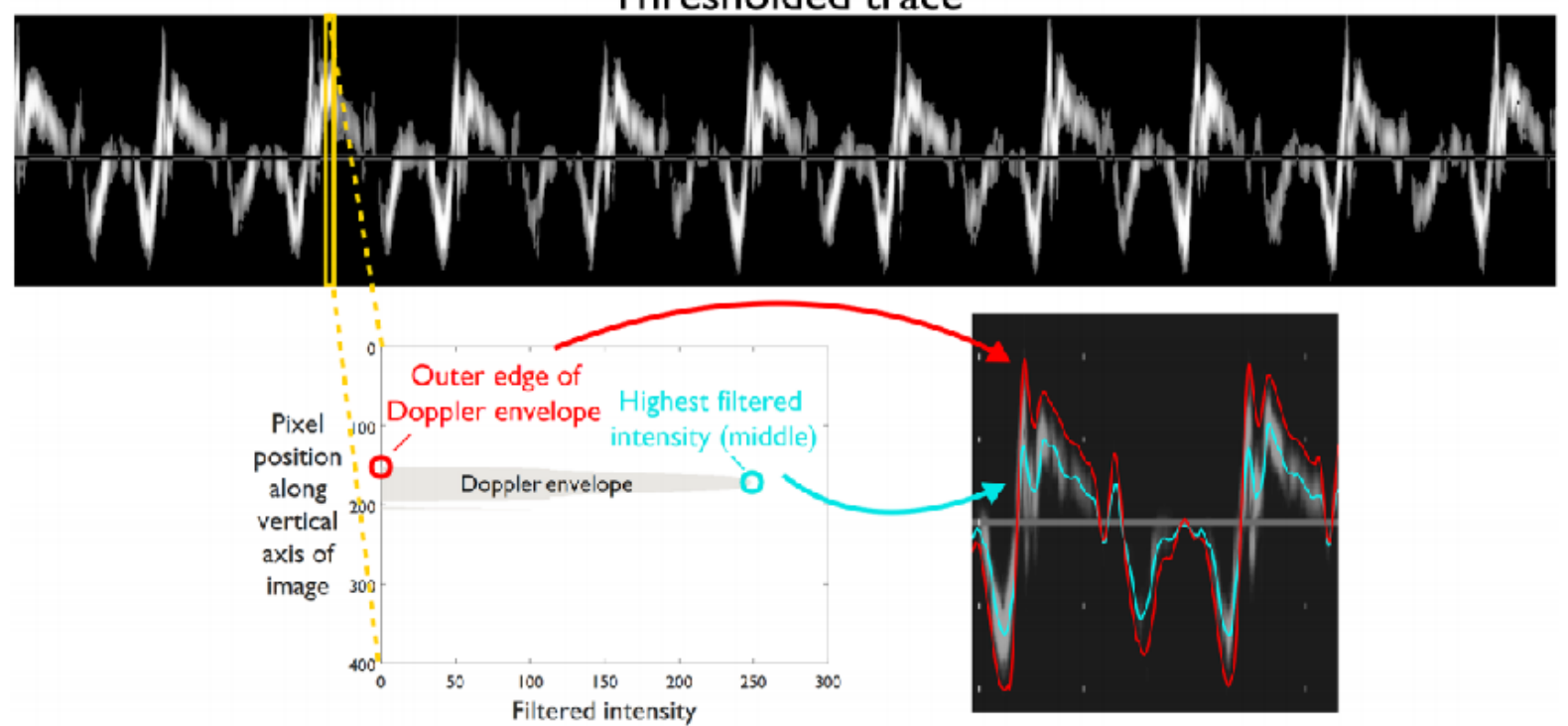

Doppler trace with automated tracing and peak detection

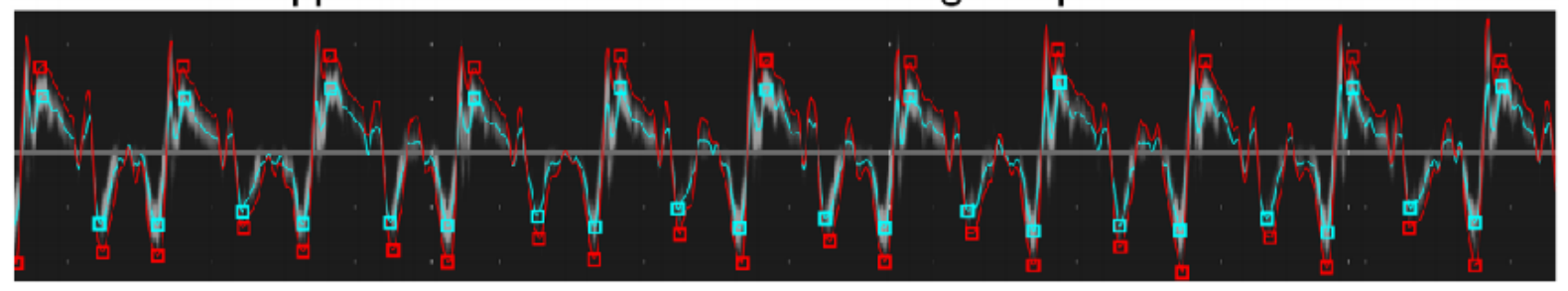

Figure 3.3: Dhutia's Doppler beats measurement (Adapted from [15])

al. [17], in 2015, proposed a motion analysis framework to classify athlete's training activities and detect potential injuries.

The athlete's data was collected by use of a variety of wearable sensors equipped with accelerometers and gyroscopes, while the athletes were performing their physical training. The proposed framework then proceeds to perform activity classification. The data was first manually segmented and annotated, using three second windows, followed by Discrete Wavelet Transform (DWT) to extract discriminative features from the accelerator data. Using the classical training / testing dataset separation, four classifiers algorithms were analysed: Random Forest, Naive Bayes, k Nearest Neighbours and Multilayer Perceptron. The Random Forest was very computationally efficient and provided the best classification, together with the use of DWT. The classifiers were evaluated with a 10 -fold cross-validation leave one out method, suit- 
able for the small dataset used. The Random Forest was able to archive a $98.3 \%$ classification accuracy.

The following step was to obtains more data of orientation and estimate joint rotation, to better analyse the motion patterns normal and injured behaviour. The orientation was extracted by a proposed algorithm, which used a gradient descent optimization to archive its results. It claimed to Kalman like results but being computationally less expensive. The joint rotation was estimated using a technique by Roetenberg et. al. [18].

The extracted data was stored in waveform, and the overall mean was obtained through an iteration update process called Procrustes method over the Squared Standard Error (SSE). The waveforms were compared using analysis of the charactering phase and statistical analysis was performed, which was able to observe significantly different data in certain parts of the activity cycle.

Although the dataset used for evaluation was very small, consisting of only nine normal subjects and one injured one, the use of DWT for feature extraction for motion data is shown to have interesting potential, as it produced a small subset of discriminating features, making it an effective and computationally efficient method.

Chen et. al. [19], in 2018, proposed a new method for classification of vessel motion pattern, in order to automatically detect anomalous and dangerous vessel movements, which can be used for accident prevention by maritime authorities.

One of the main challenges with analysing vessel motion is the amount of data to process, the lack of complete labelling and missing / incomplete data on the vessels motion. For this type of problem, the author used a Sparse Representation Classification (SRC) method, a novel classification technique, which has shown good results even with incomplete training data, making it suitable for the studied dataset.

Using the SRC method presents a challenge in that at its core it's a L0-minimization problem, which is a NP-Hard problem [20], requiring approximation and heuristics methods to make the classification computationally feasible. In this case, many studies have changed the L0minimination into a L1-minimization or used convex optimization solvers, enabling a polynomial solution to the problem.

To solve the SRC problem in this vessel motion data, the author proposed a new method, the Matching Pursuit - Fletcher Reeves, which is adequate for non-convex problem.

Prior to the classification, a motion profile dictionary is built, splicing the motion trajectories into fixed-length objects. For this purpose, the Least-squares Cubic Spline Curves Approximation (LCSCA) technique was used to simplify the motion trajectories curves, with the b-spline controls points being used to represent shape and space-temporal profiles. Figure 3.4 demonstrates this study's framework.

The study compared the results of six different classifiers, Support Vector Machine (SVM), k-Nearest Neighbor (k-NN), SRC with I1 normalization, SRC with I2 normalization and SRC with Matching Pursuit - Fletcher Reeves normalization. The proposed method presented the 
best results in terms of accuracy and True Positive Rate (TPR) and False Positive Rate (FPR).

The added complexity of the proposed method yielded a small gain of $3 \%$ over a simpler generic k-NN method, and the algorithm was formulated for this specific problem, making it a more domain specific solution. In this case, considering the volume of data and interest in the solution for monitoring and other purposes, justifies the development of such a specialized solution.

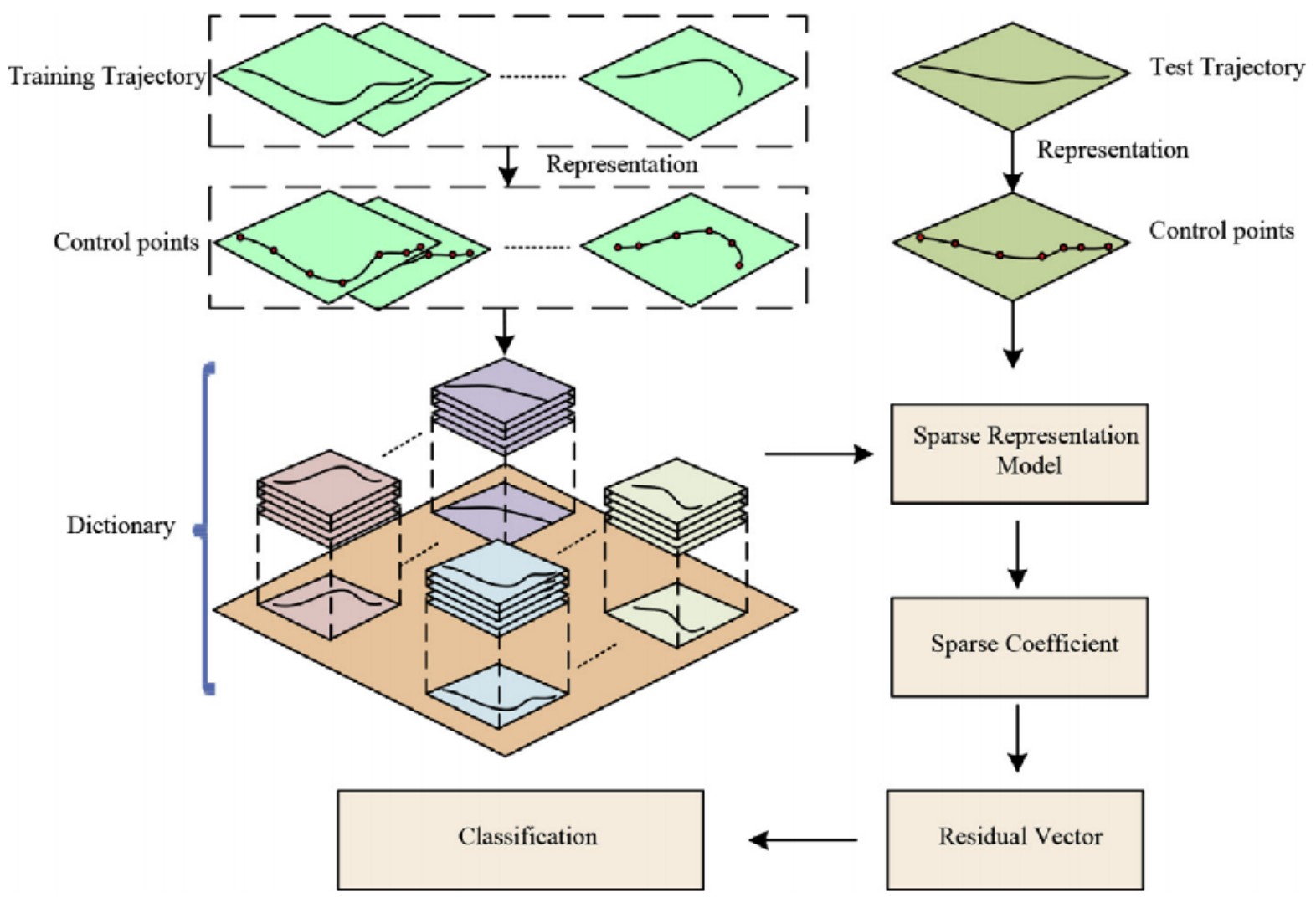

Figure 3.4: Chen's proposed framework (Adapted from [19])

\subsection{Discussion}

The mentioned studies all contain interesting proposals in the fields of automatic measuring and motion analysis. The presented concepts influenced on the development of the proposed methods, such as how N. Subramanian et. al. used orthogonal lines. Ahmadi et. al. wavelets usage to extract features which were then used for statistical classification, served as a conceptual starting point for the proposed motion analysis method, that while not using wavelets, used a mathematical competitor to it, to extract features for classification.

Dhutia et. al. lead to ideas on how to extract information about the cardiac cycle, to improve the motion analysis. While this approach could not be used on the used dataset, as it lacked the 
Doppler beats data, the proposed motion analysis method if used together with this extracted data, could lead to significant improvements in overall results.

Chen et. al. proposal to use LCSCA technique to simplify motion was evaluated to be used on the motion analysis method, but the motion data extracted from the dataset was not considered to need another step of simplification. The prosed use of SRC was demonstrated to yield small gains for the added complexity, so it was not used. 


\section{Chapter 4}

\section{Thickness Profile Extraction}

\subsection{Introduction}

The WHF guidelines criteria defines the valve thickening as a level 2++ evidence [3], identifying it as a very important characteristic for diagnosis of RHD. An AML's tip 3mm cut-off criteria for school-age children has been recommended, as the peak incidence of acute rheumatic fever is at the age of 5 to 15 years old [21].

This criteria is for measurements done during the diastolic phase, in the parasternal longaxis (PLAX) view [3]. This is due to the fact that in the diastolic phase the AML is open, avoiding merging with other regions like the chordae tendineae or the posterior mitral valve leaflet, which can interfere with the measurement.

Manual Thickness measurement has issues as it's a process very prone to inter and intra observer variability duo to the low image quality of two-dimensional echocardiogram, with poorly defined edges, and lack of properly specified measurement locations. The WHF guidelines [3] only indicate a thickness threshold at the tip, but no precise indication of how this measurement should be made, leaving this to a physician's highly variable interpretation.

Given the importance of AML thickness as a clinical decision factor of RHD diagnosis, and the typical subjectivity and difficulty of manual measurements, this chapter aims to propose an objective methodology to automatically obtain the thickness profile of the AML, based on its previously segmented contour.

\subsection{Materials}

An initiative from the Real Hospital Português, in Recife, Brazil led to the screening of 1203 children and pregnant women, searching for cardiac pathologies. Data was collected using different ultrasound devices (Vivid i model by GE healthcare and CX50 model by Philips), with a wide range of transducers, frequency and scanning depths. Depending on the equipment and depth, the spatial resolution ranged between 0.115 to $0.384 \mathrm{~mm}$. Forty of these exams 
were manually annotated by doctors using the OsiriX software and were used to extract the thickness profiles studied in this thesis.

The annotated dataset contains eight pathological cases and thirty-two normal cases. These forty videos include a total of 3825 frames with dimensions of $422 \times 636$ pixels and all videos are of the Parasternal long-axis (PLAX) view. Framerate ranged from 30 frames per sec to 120 frames per sec. On average each video consists of two or three seconds of content. The videos also contained metadata from the exam, such as resolution, frame rate, etc.

The rotation of the probe, resolution and frame rate used in this dataset varied by a large margin, so any algorithm used to analyse the data had to be robust against these conditions.

This proposed method has been implemented using $\mathrm{C}++17$ on a portable computer (QuadCore Intel i7-3630QM processor, 2.4 GHZ, 16 GB RAM) in a Windows 10 and Linux environment. The data analysis and graph plotting were done in $\mathrm{R}$ 3.4.3.

\subsection{Framework}

A framework was defined to measure and analyse the thickness of the AML, as shown on Figure 4.1.

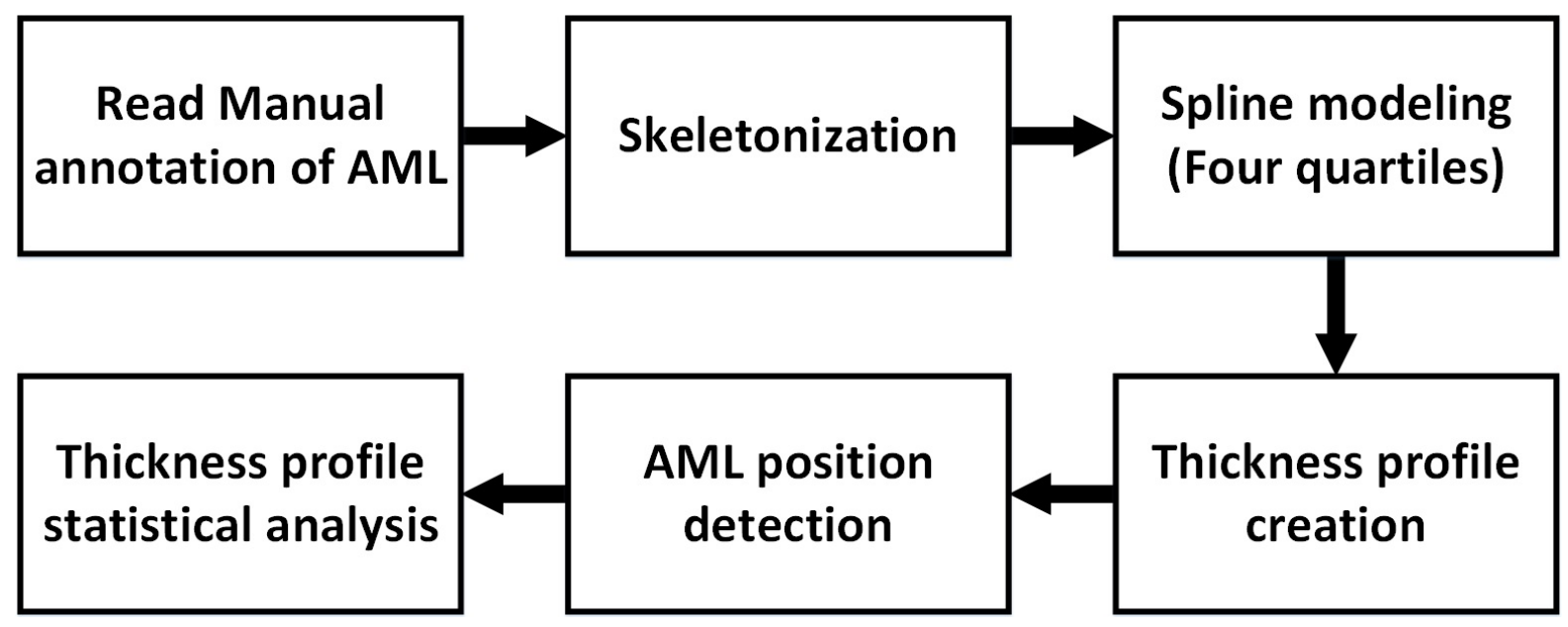

Figure 4.1: The Thickness Profile Extraction Framework

The annotation of the AML contour used, was manually done by doctors, allowing it to avoid the contamination of errors originated by automatic segmentation algorithms, and thus enabling it to confirm if the thickness is indeed an important feature to identify early RHD cases.

After reading the manually annotated data, the shape of the AML is simplified by a skeletonization process, followed by spline modelling and by the division of this line into four quartiles. The position of the leaflet is also estimated (open, straight, closed) in order to better understand what the best position is to measure the thickness profile. The final step is the thorough analysis of the data to define proper recommendations based on the statistical evidence. 


\subsubsection{Skeletonization}

As the AML's shape is complex and uneven, making it difficult to establish reproducible landmarks, a skeletonization process was first applied to this shape.

Several algorithms were considered for this step. The more traditional Open and Close morphological operations, while computationally very efficient failed to produce anatomically correct results. Thinning techniques used for applications of pattern recognition of hand writing proved to yield interesting results, as the AML's shape is quite similar to uneven strokes, possibly motivating and justifying its interesting mapping from an alphanumeric skeletonization scenario to a physiological structure one, as exemplified in Figure 4.2.
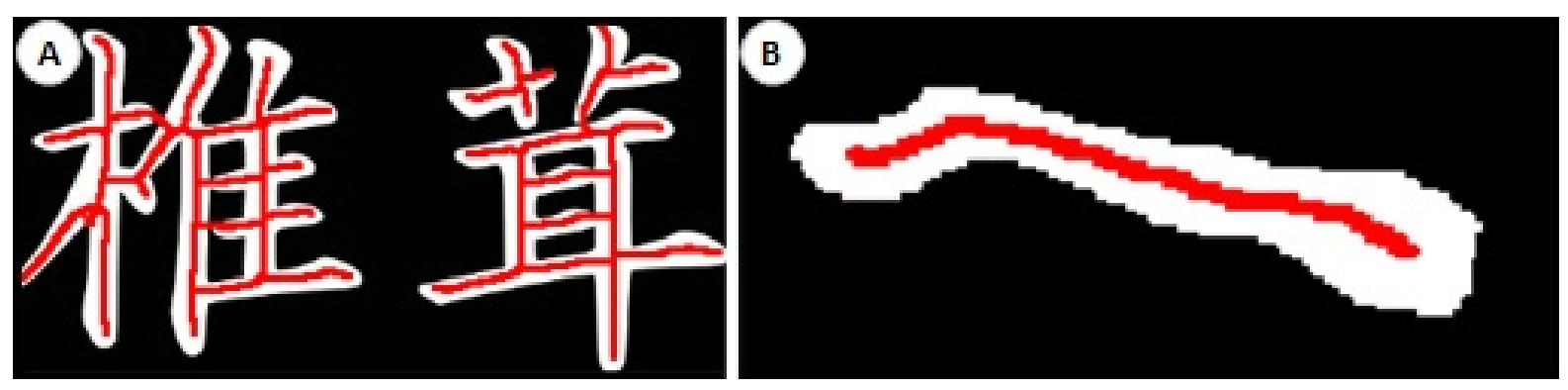

Figure 4.2: A) Skeletonization of chinese character B) Skeletonization of the anterior mital leaflet

The first algorithm analysed was the Zhang-Suen thinning algorithm [22], proposed in 1984. The proposed algorithm presumes that the input data is a binary image matrix, and that each pixel is surrounded by eight pixels in a $3 \times 3$ matrix, which means it cannot be used on the image's corners. Figure 4.3 demonstrates the $3 \times 3$ matrix mapping around the point $(\mathrm{i}, \mathrm{j})$, used by the algorithm.

\begin{tabular}{|c|c|c|}
\hline$P_{9}$ & $P_{2}$ & $P_{3}$ \\
$(\mathrm{i}-\underline{\underline{1, j}-1)}-1)$ & $(\mathrm{i}-\underline{\underline{1}, \mathrm{j})}$ & $\underline{\underline{1}, \mathrm{j}}+1)$ \\
\hline$P_{8}$ & $P_{1}$ & $P_{4}$ \\
$(\mathrm{i}, \mathrm{j}-1)$ & $(\mathrm{i}, \mathrm{j})$ & $(\mathrm{i}, \mathrm{j}+1)$ \\
\hline$P_{7}$ & $P_{6}$ & $P_{5}$ \\
$(\mathrm{i}+1, \mathrm{j}-1)$ & $(\mathrm{i}+1, \mathrm{j})$ & $(\mathrm{i}+1, \mathrm{j}+1)$ \\
\hline
\end{tabular}

Figure 4.3: Zhang-Suen Neighbor Pixels Mapping

The algorithm does two iterations. On the first iteration, the point $P_{1}$ is deleted if it satisfies all of the following conditions:

a The number of non-zero neighbours is between two and six

b Number of 01 patterns if equal to 1 , as exemplified in Figure 4.4 
c $P_{2} * P_{4} * P_{6}=0$

d $P_{4} * P_{6} * P_{8}=0$

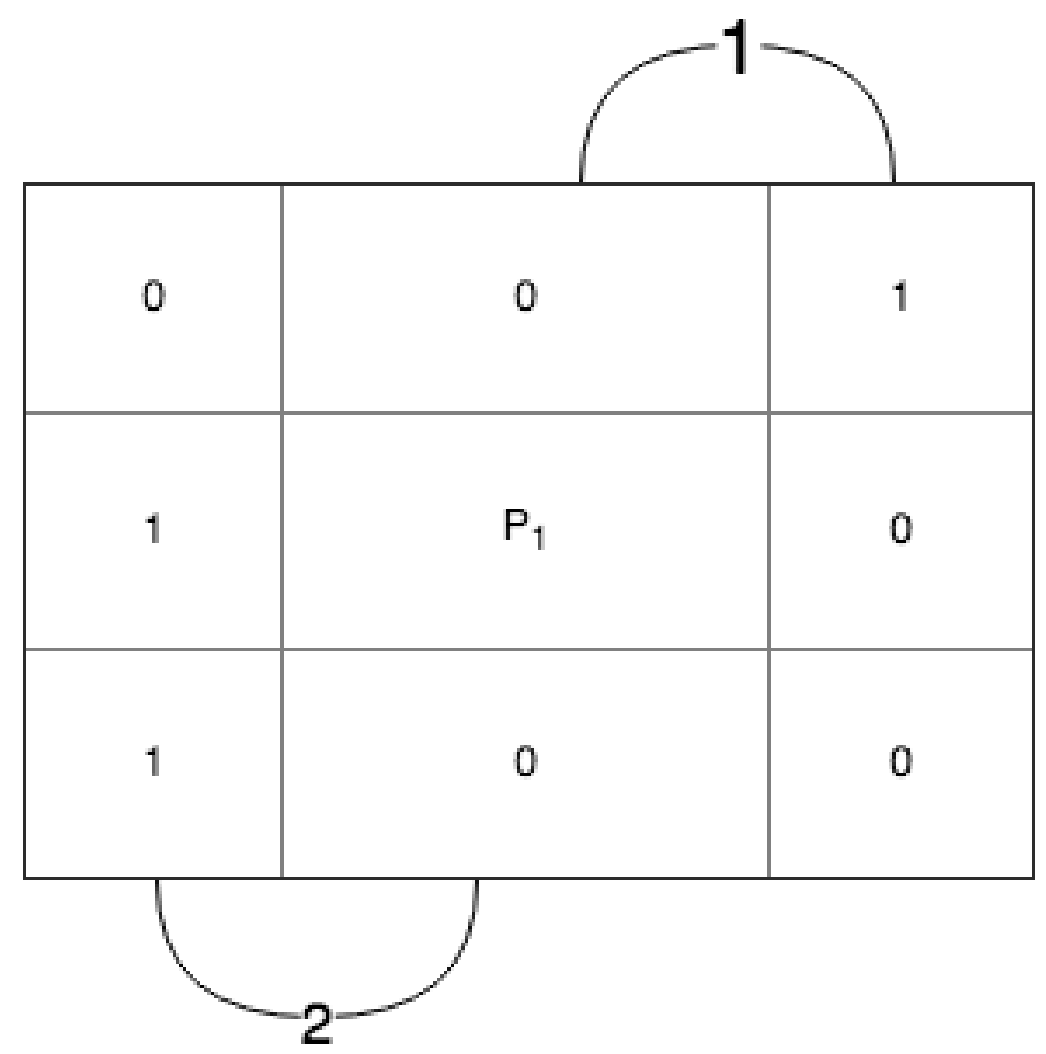

Figure 4.4: Zhang-Suen 01 Pattern Example

On the second iteration, condition $a$ and $b$ remain the same, while conditions $c$ and $d$ change into the following:

c $P_{2} * P_{4} * P_{8}=0$

d $P_{2} * P_{6} * P_{8}=0$

The first iteration only removes the south-east points that should not belong to the skeleton, while the second iteration removes the north-west points, all while preserving the endpoints. This process is repeated until there are no more points left to remove.

The algorithm obtained excellent results, compared to the traditional morphological operations, in maintaining connectivity and shape representations of the AML, while being computationally inexpensive. The two-iteration system allows for easy parallelization, enabling an even greater speedup on modern multi-core CPUs.

A variation by Zhang-Wang [23] was also tested, but yielded no meaning differences to the Zhang-Suen algorithm [22] in terms of results and performance, while requiring a larger border 
due to the $4 \times 4$ matrix used. More recent thinning algorithms were also explored, such as KMM [24] and in particular an improved version of it called K3M [25].

$\mathrm{K} 3 \mathrm{M}$ also works using a $3 \mathrm{X} 3$ matrix for each binary pixel. It however performs a set of six sequential iterations, repeated until it matches the condition to perform a last 1-pixel thinning step.

The K3M uses lookup arrays to decide which pixels to remove. The iterations behaviour is the following:

1. Mark the border pixels to be analysed

2. Deletes the border pixels that have 3 neighbours

3. Deletes the border pixels that have 3 or 4 neighbours

4. Deletes the border pixels that have 3,4 or 5 neighbours

5. Deletes the border pixels that have $3,4,5$ or 6 neighbours

6. Deletes the border pixels that have $3,4,5,6$ or 7 neighbours

7. Unmark the border pixels left

8. If any pixel was modified in this iteration, return to step 0.

9. Deletes the border pixels matching the final 1-pixel width lookup array

This process is defined in more detail in Figure 4.5.

The last step was defined by the authors based on multiple experiments to obtain the most versatile lookup array.

The K3M algorithm sequential nature makes it non-trivial to parallelize, requiring the splitting of the scan area into multiple windows, forcing us to deal with the windows edge's race condition. Using mutexes, semaphores or atomic variables could potentially overcome these limitations, but as K3M quality gains over the Zhang-Suen algorithm were marginal for the AML's skeletonization and its simple single core computational cost was higher, the ZhangSuen algorithm was selected, as it provided excellent results while being computationally very efficient, enabling real-time usage.

\subsubsection{Spline Modelling}

Due to the uneven shape of the AML, the previous skeletonization process can produce branches that would normally require trimming to produce a smoother skeleton. The produced skeleton also still exhibits important sudden variations in its first derivative, making the calculation of its normal an unstable process, quite sensitive to the exact choice of the point of the 

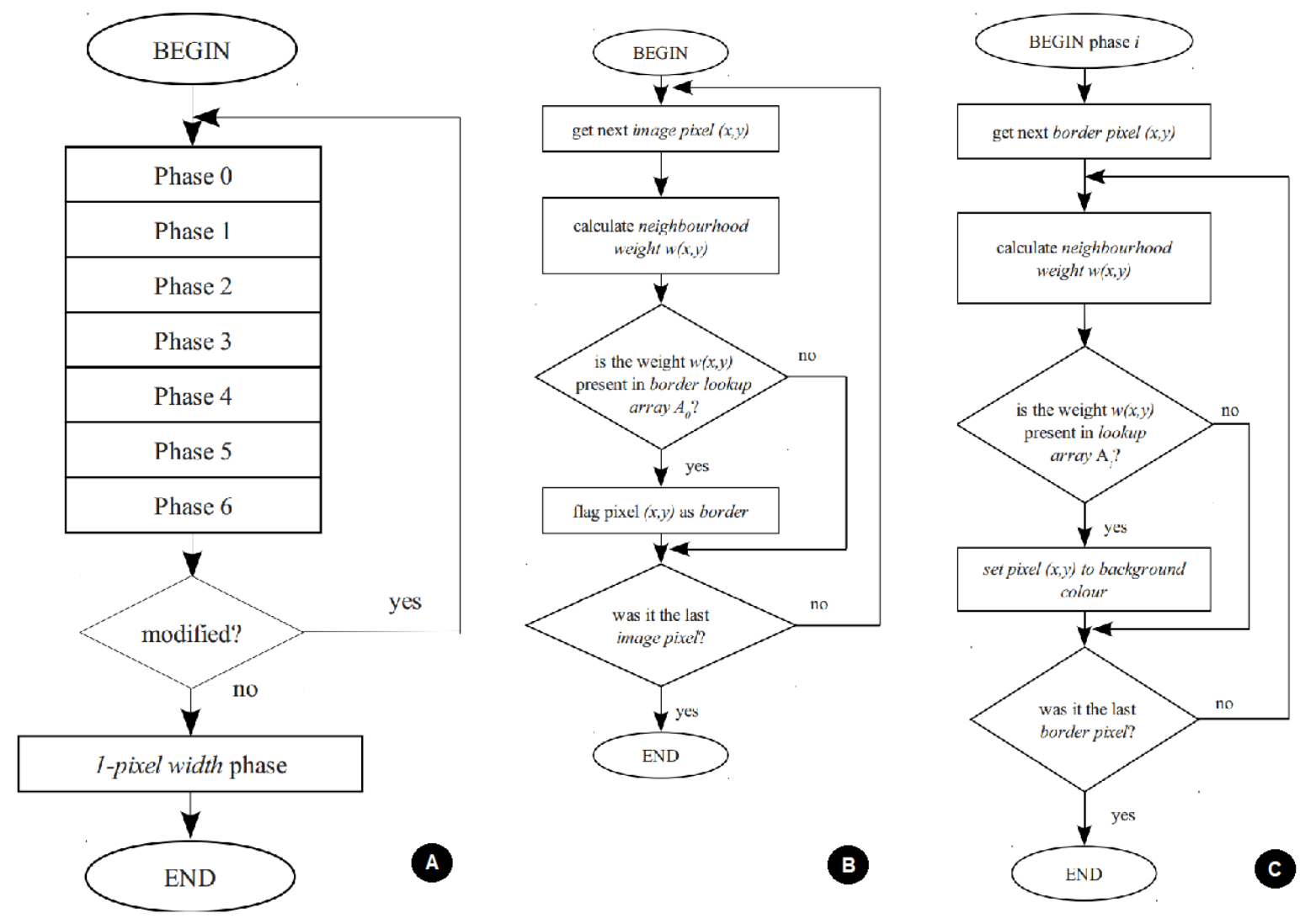

Figure 4.5: A) K3M Simplified Flowchart B) Phase 0 C) Phase i $(i \in[1,2,3,4,5])$ (Adapted from [25])

line intersected by this normal. The calculation of the normal is necessary for the thickness measurement of the AML, so it's stability is critical to produce stable and reproducible results.

Cubic interpolation is a mature [26] and widely used solution for interpolation and smoothing image data. It behaves well numerically producing smooth curves and as the lowest polynomial that supports inflection it's very computationally efficient. Since splines are piecewise, not depending on a single formula / function, several types are available. To define the appropriate one, the following requirements were set:

- Continuous - Smooth without breaks or jumps on the function. Important as the skeleton cannot have any breaks. The first derivative of the function should also be continuous, as sharp directional changes are undesirable for skeleton's shape.

- Interpolated points must pass through the defined control points.

- Low computational cost

Catmull-Rom Spline [27] didn't meet the first requirement, and the B-Spline (Basis Spline) [28] failed to meet the second. Natural Spline [29] met all requirements, and possesses an 
additional characteristic: lack of local control, meaning that the shift of one control point, would cause a change in the entire spline. In practical tests this proved to be a desirable feature as the end result was stable and had a higher fidelity to the original skeleton's shape.

To define which control points to use when calculating the spline, the pixels of the skeleton where first sorted using Quicksort [30], which provides good performance and low memory consumption, according to their $X$ and $Y$ positions. This is done by using the $X$ position as the primary comparison for sorting and the $Y$ position as a secondary comparison for when the comparing pixels possess the same $X$ position. This adaptation allows the algorithm to sort using both axis in only one pass. On this sorted pixel list, five evenly spaced points were selected, ranging from the extremities of both ends of the AML, thus forming four equally sized sections henceforth referred to as quartiles labelled Tip, Mid-1, Mid - 2 and Tail.

These selected points were then used as the controls points of the Natural Spline [29], forming a smooth, stable and branch free skeleton. Figure 4.6 illustrates this process.

\subsubsection{Thickness Measurements}

The spline modelling, which replaced the original skeleton, represents a simplified central line of the AML. The AML's shape is defined by the boundaries of the binary mask, obtained by using an edge detection algorithm [31] on the binary annotated data.

Using the mid-point of each of the spline's quartiles, as defined on the previous section, the associated normal is calculated, creating intersection points between the central line and the AML's shape contour. The distance between these intersection points corresponds to the thickness measurement for each quartile, which is converted from pixels to millimetres based on spatial resolution parameters obtained from the metadata present in the video files. Figure 4.7 demonstrates the final result.

\subsubsection{Position Detection}

As the information about the AML's position in each frame (open, straight, closed) was not provided in the manual annotation of the dataset, a simple automatic classifier was built to compute this information, providing a rough initial classification that was later validated and corrected by an expert.

This simple classifier algorithm compares the angle between a perpendicular line through the tail point and a line passing from the tail point through the tip point. The classifier uses the following rules:

- If the angle is greater than $170^{\circ}$ and less than $190^{\circ}$ - Position Straight

- If the angle is less than $170^{\circ}$ - Position Open

- If the angle is greater than $190^{\circ}$ - Position Closed 

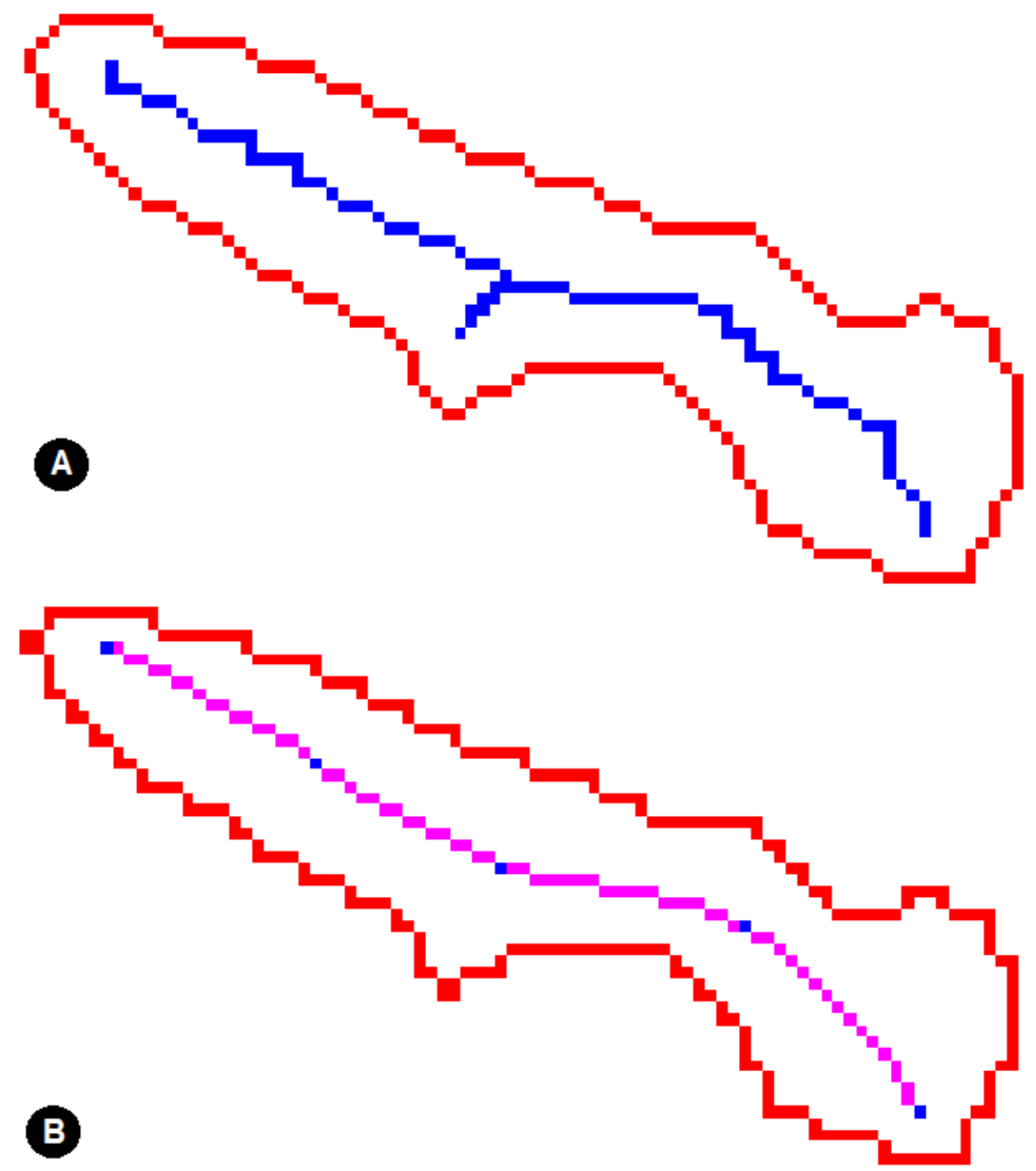

Figure 4.6: A) Original Skeleton B) Natural Spline of the Skeleton

The classifier also calculates a confidence level for the classification based on the distance from the angle threshold, as well the mean and variance of the $Y$ value of the spline control points. This extra information is used by the expert to filter which automatic classifications are more likely wrong, speeding up the manual review process.

\subsection{Results}

The dataset was processed with the developed framework, providing data, which was the used to profile the AML's thickness. This statistical data allowed for verification and validation of the WHF guidelines [3] as well as new observations. 


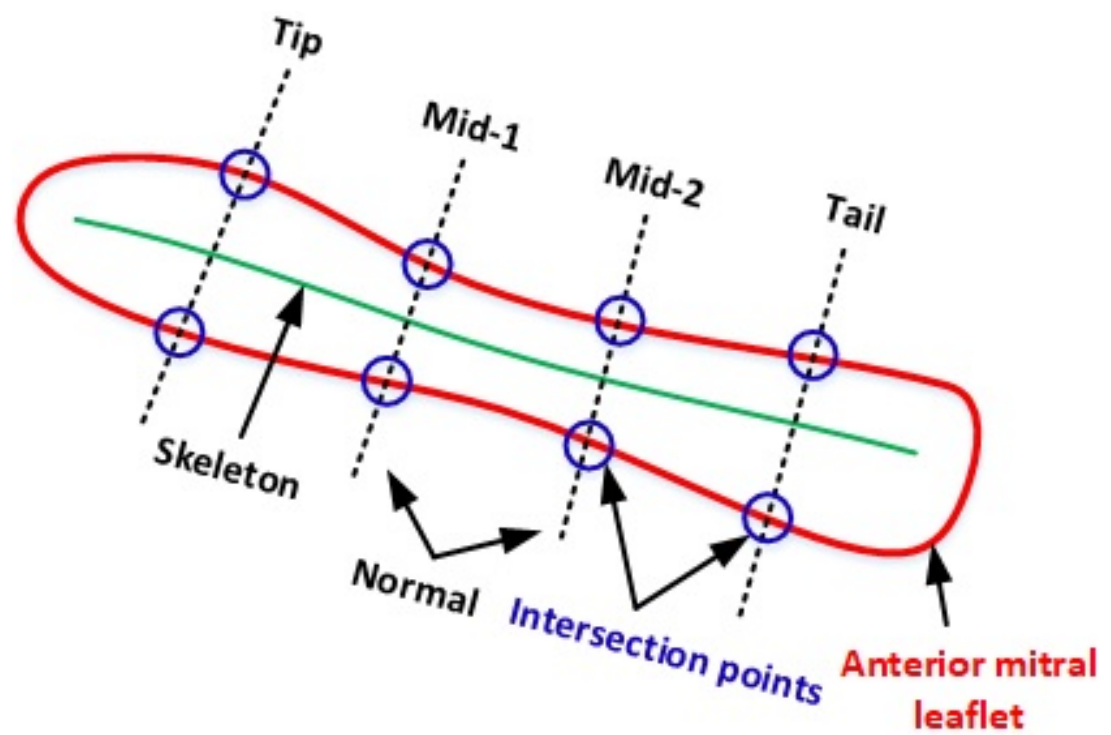

Figure 4.7: Thickness Measurements

\subsubsection{Average Thickness by Quartile}

Figure 4.8 uses a box plot to depict the analysis of the thickness profile for each quartile of the whole dataset. It shows the general distribution of the AML's thickness. The median thickness of the quartiles was found to be the following:

- Tip $-2.8 \mathrm{~mm}$

- Mid-1-2.0 mm

- Mid $-2-2.2 \mathrm{~mm}$

- Tail - $3.1 \mathrm{~mm}$

This demonstrates that the Tip and Tail are on average thicker than the Mid -1 and Mid -2 . The $p$-values between all the quartiles measurements were less than 0.05 , indicating a statistical difference, establishing that the measurements were taken in adequate locations for thickness profiling. The data demonstrated by the Box Plot provides information of the thickness distribution per quartile, providing guidelines to the overall shape of the leaflet and how it changes from Tip to Tail.

\subsubsection{Normal Versus Pathological Thickness Profiles}

Following the recommendation of the WHF guidelines [3], a statistical analysis was performed to verify if the Tip is thicker in pathological cases than in normal ones. For completeness, it was also analyzed how the $M i d-1, M i d-2$ and Tail thickness vary between normal and pathological cases. Figure 4.9 clearly shows how the thickness correlates with the 


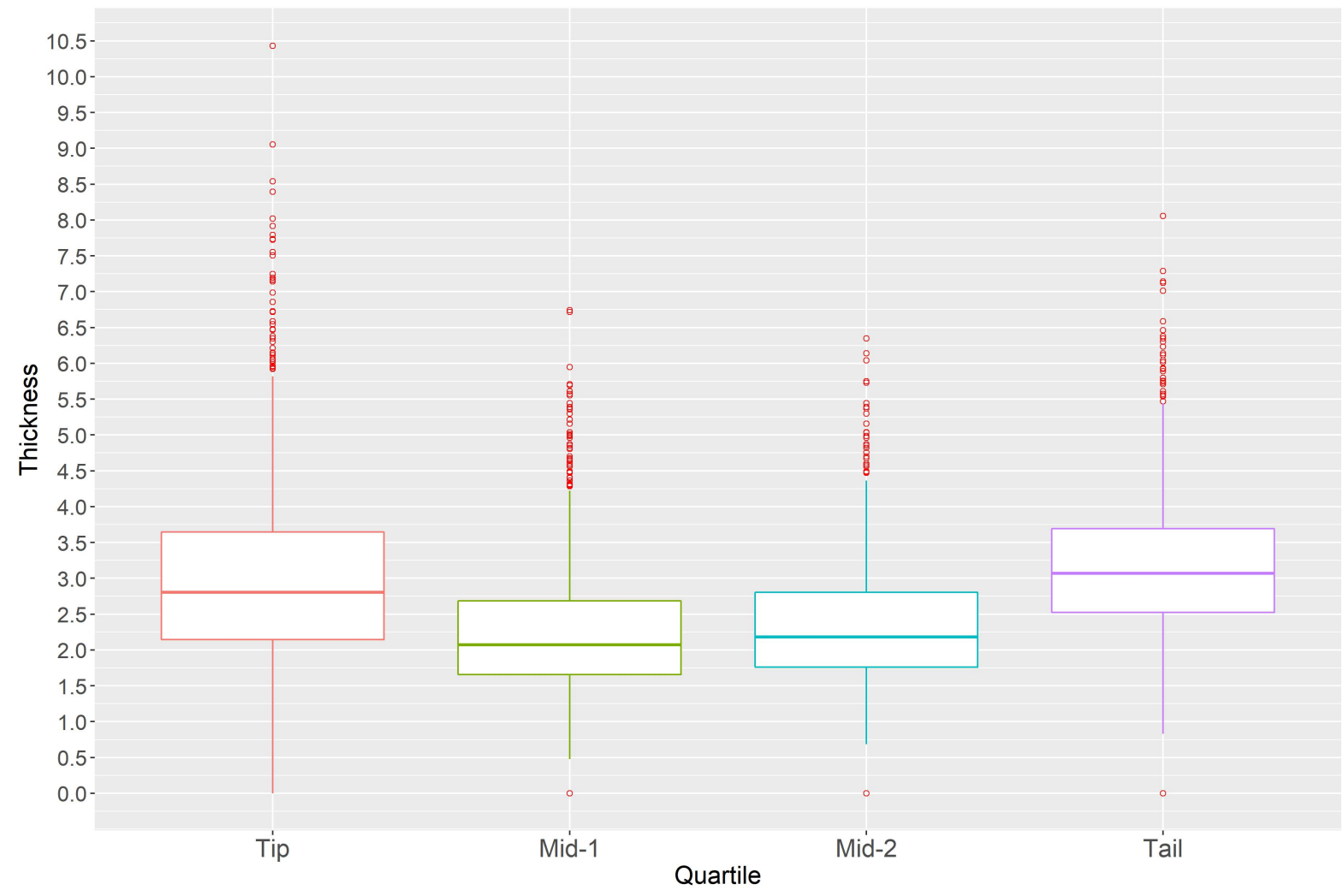

Figure 4.8: The thickness profile of the Anterior Mitral Leaflet

pathology. In pathological cases the thickness of the whole leaflet is significantly larger when compared with normal cases. The median thickness increases in pathological cases at the Tip, Mid-1, Mid -2 and Tail, as indicated on Table 4.1. is respectively $0.957 \mathrm{~mm}, 0.534 \mathrm{~mm}$, $0.147 \mathrm{~mm}$ and $0.215 \mathrm{~mm}$.

\begin{tabular}{c|ccc} 
Quartile & Normal & Pathological & $\varnothing$ \\
\hline Tip & 2.638 & 3.595 & 0.9575 \\
Mid -1 & 1.983 & 2.517 & 0.5335 \\
Mid -2 & 2.182 & 2.329 & 0.1472 \\
Tail & 3.022 & 3.237 & 0.2158
\end{tabular}

Table 4.1: Median Thickness

The WHF criteria is well demonstrated for the Tip, showing that the most frequent thickness values stay above the defined threshold of $3 \mathrm{~mm}$ for the pathological cases. Another observation was the increase in spread of the most frequent thickness values (1st to 3rd quartiles) in the $M i d-1$ section in the pathological cases. The criteria of measuring the thickness only during the Straight position should be further investigated, as no statistical difference was found between the Open and Straight position's thickness on pathological cases. 


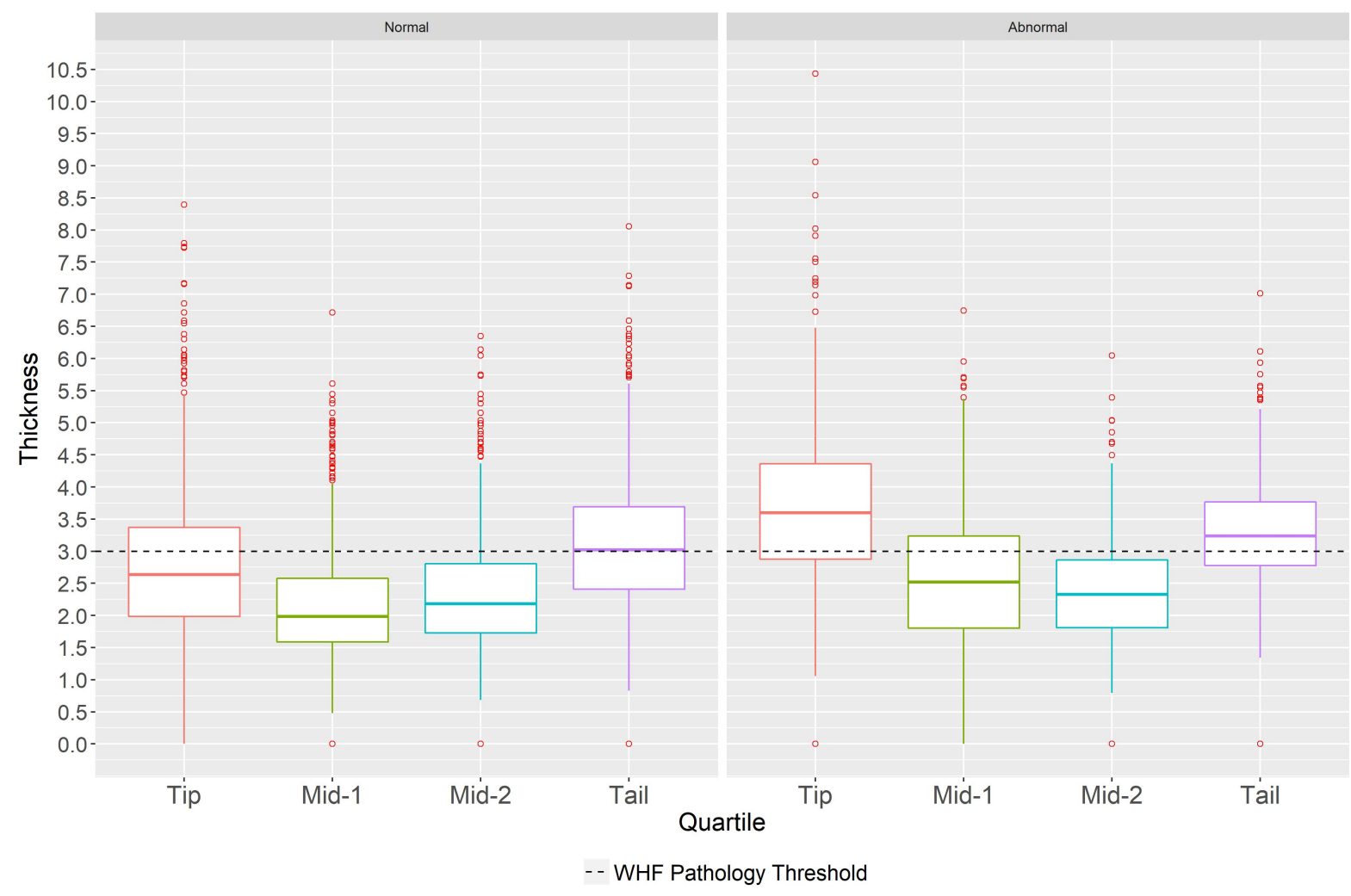

Figure 4.9: The thickness profile of the AML by Normal vs. Pathological cases

\subsubsection{Quartile Versus Position}

The thickness measurements for normal and pathological cases were analyzed relative to the position of the AML (open, closed and straight). The values presented on Table 2 validate the statistical significance difference between normal and pathological cases when measuring the thickness at the tip of the AML, as it is also confirmed by running several nonparametric Friedman tests on random samples and doing a Bonferonni correction on the respective pvalues.

Table 4.2 is composed of the median p-values and the median difference between the pathological vs. normal values at each AML position and each quartile. It indicates that measuring of the thickness of the Tip can be done in all the positions. As the thickness values for the pathological cases on the closed position are statistically different from straight and open, the WHF threshold $(3 \mathrm{~mm})$ usage is limited to the Open and Straight position. It can also be seen that quartile $M i d-2$ 's thickness does not exhibit statistically significant difference and thus it should not be recommended as a criterion to determine the presence of a RHD pathology. 


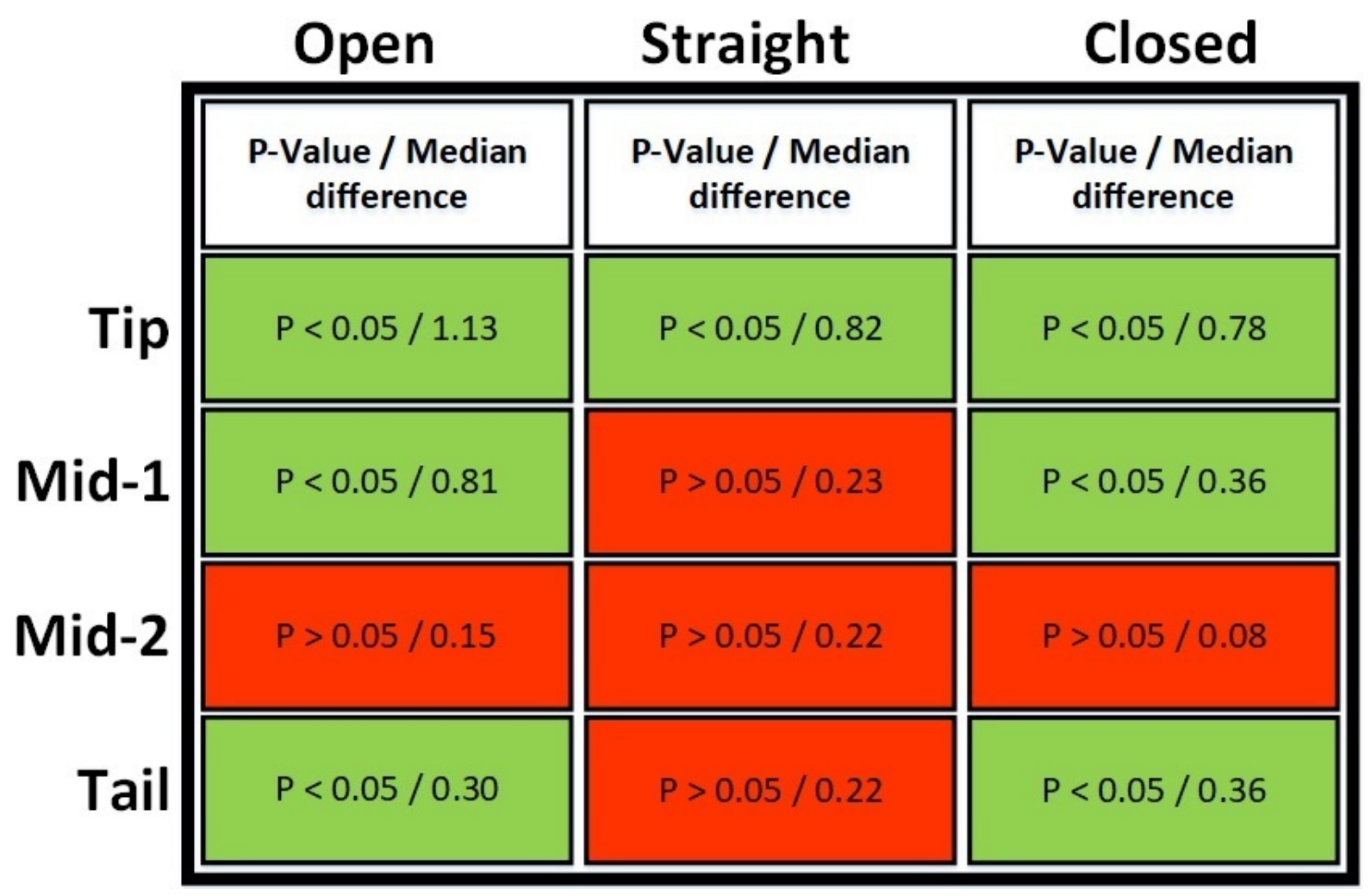

Table 4.2: Thickness Variance Between Pathologies

\subsection{Discussion}

The proposed method produced statistically significant data regarding the leaflet's thickness in different quartiles, positions and normal and pathological cases, providing information on its overall shape and its variance. It provided further evidence of the leaflet's thickening on pathological cases.

It was also observed that the leaflet's tip measurements on normal, open and closed positions provided statistical significant difference, indicating that the measurements could be performed in all the positions, although on the closed position it would not be recommended due to the fact that segmentation on this area poses a challenge as the proximity between the AML and Posterior Mitral Valve leaflet can lead to over segmentation of the AML and an incorrect tip placement. 


\section{Chapter 5}

\section{Motion Classification}

\subsection{Introduction}

The WHF guidelines [3] classifies both excessive and restricted leaflet tip motion as level $2++$ evidence of RHD. This demonstrates the significance that the AML motion analysis has, motivating us to research novel algorithms for this automatic classification.

Excessive leaflet tip motion is caused by either the elongation or the rupture of the primary chords [32], while restricted leaflet tip motion is caused by the shorting or fusion of the primary chords, the leaflet thickness, calcification or commissural fusion [3]. Retraction of the secondary chordae can cause a V-shaped deformity in the central area of the AML, causing restricted motion movement [32].

The proposed method to perform motion classification used elements of the proposed Thickness Profile Extraction method described in Chapter 4 within a pattern recognition framework. It aims to extract relevant features based on the segmented data motion patterns and use those to perform automatic classification of RHD, potentially aiding the physician on the screening process.

\subsection{Materials}

An initiative from the Real Hospital Português, in Recife, Brazil led to the screening of 1203 children and pregnant women, searching for cardiac pathologies. Data was collected using different ultrasound devices (Vivid i model by GE healthcare and CX50 model by Philips), with a wide range of transducers, frequency and scanning depths. Depending on the equipment and depth, the spatial resolution ranged between 0.115 to $0.384 \mathrm{~mm}$. Forty of these exams were manually annotated by doctors using the OsiriX software and were used to extract the thickness profiles studied in this thesis.

The annotated dataset contains eight pathological cases and thirty-two normal cases. These forty videos include a total of 3825 frames with dimensions of $422 \times 636$ pixels and all videos 
are of the parasternal long-axis (PLAX) view. Framerate ranged from 30 frames per sec to 120 frames per sec. On average each video consists of two or three seconds of content. The videos also contained metadata from the exam, such as resolution, frame rate, etc.

The rotation of the probe, resolution and frame rate used in this dataset varied by a large margin, so any algorithm used to analyse the data had to be robust against these conditions.

This proposed method has been implemented using $\mathrm{C}++17$ on a portable computer (QuadCore Intel i7-3630QM processor, 2.4 GHZ, 16 GB RAM) in a Windows 10 and Linux environment. The data analysis and graph plotting were done in R 3.4.3.

\subsection{Framework}

A framework was defined to extract motion features of the AML and perform pathology classification, as shown on Figure 5.1.

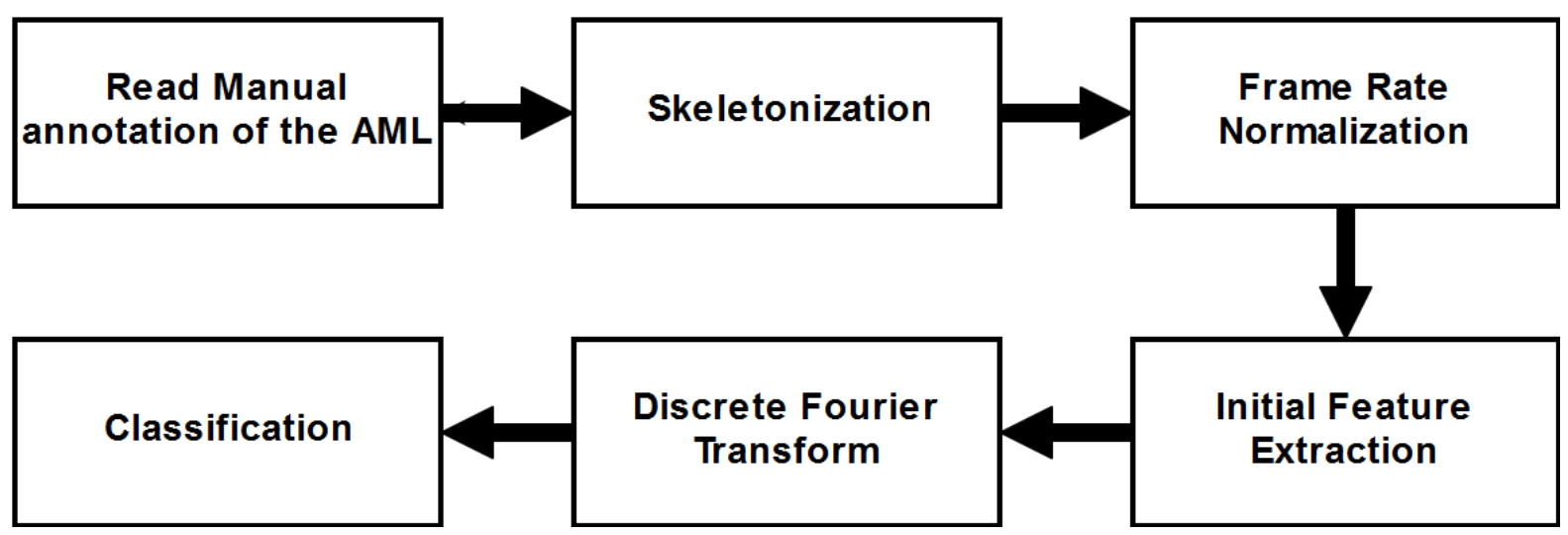

Figure 5.1: The Motion Classification Framework

The annotation of the AML contour used, was manually done by doctors, allowing it to avoid the contamination of errors originated by automatic segmentation algorithms, avoiding introducing errors into the dataset.

After reading the manually annotated data, the shape of the AML is simplified by a skeletonization process, where five control points are extracted. The frame rates of the videos on the dataset are normalized prior to the extraction of the initial features. This is done by using the analysis of the motion of these points across the frames of the echocardiogram videos. The Discrete Fourier Transform (DFT) is then applied to these initial features, producing the feature dataset used by the final classification step.

\subsubsection{Skeletonization}

The skeletonization method from the proposed method of Thickness Profile Extraction was used, as described in Chapter 4, but the spline modelling and thickness measurements steps were not performed. Due to the fact that only the motion of the control points was going to be 
analysed in this method, the steps that were used to extract thickness data, spline modelling and thickness measurements, were unnecessary. By eliminating these steps, a reduction in the computational cost of this operation was achieved.

\subsubsection{Initial Feature Extraction}

In order to build a classification model for the AML's motion, it was necessary to extract comparable features that represented such motion. Using the simplified skeletonization process, several features were extracted from the dataset.

- Horizontal Velocity - $\mu \mathrm{m}$ per second

The calculated velocity between frames on the horizontal axis for each control point

- Vertical Velocity - $\mu$ m per second

The calculated velocity between frames on the vertical axis for each control point

- Absolute Velocity - $\mu \mathrm{m}$ per second

The absolute velocity between frames for each control point calculated by using the Euclidian distance

- Depth Velocity - $\mu \mathrm{m}$ per second

The velocity between frames for each control point of their depth change

- Acceleration - $\mu \mathrm{m}$ per second

The acceleration between frames for each control point - Calculated from the derivative of the Absolute Velocity

- Arc Tangent - Radians

The angle of the direction of the motion calculated by using the arc tangent

The Horizontal, Vertical and Absolute Velocity were the basic motion features to be analysed on the leaflet's motion patterns. The depth velocity was extracted to obtain the velocity of the depth variation, which has a partially fixed point: the echocardiogram's beam origin point, even though as it's still subjection to the operator's tremors and motion, but to a small degree as the extracted sample is of a short period of time - one second.

Acceleration was extracted on the basis that the second derivative might better represent the sudden changes on the leaflet's motion patterns compared to the first derivative. The direction angle was calculated using the arc tangent function to observe the directionality of the leaflet's motion, to better understand the more complex motion patterns of it. 


\subsubsection{Frame Rate Normalization}

The videos on the dataset had different frame rates, ranging from thirty frames per second to a hundred and twenty frames per second. In order to make the extracted features comparable and suited for the next steps of processing, it was necessary to homogenize the frame rates. It was decided to perform temporal subsampling to match the lowest frame rate value on the dataset, thirty frames per second.

The manually annotated data on the dataset had missing information on a few random frames, duo to the low image quality, which prevented the physician from properly segmenting the AML's location. As such, the frames which contained no manually annotated data were not utilized for the analysis. Interpolation was not considered a viable option as in certain videos there were several consecutive frames without annotated data, and thus the interpolated data would have too much noise, which could potentially worsen the results of the motion classification.

The defined method was to use blocks of one second of continuous annotated data and perform the motion analysis on each of those blocks. The temporal subsampling process performed on these blocks made the distance measurements taken afterwards comparable as time elapsed between the frames would then be equivalent.

To perform this temporal subsampling and extract the mentioned features automatically, an algorithm was developed. The pseudocode 1 demonstrates this algorithm's behaviour.

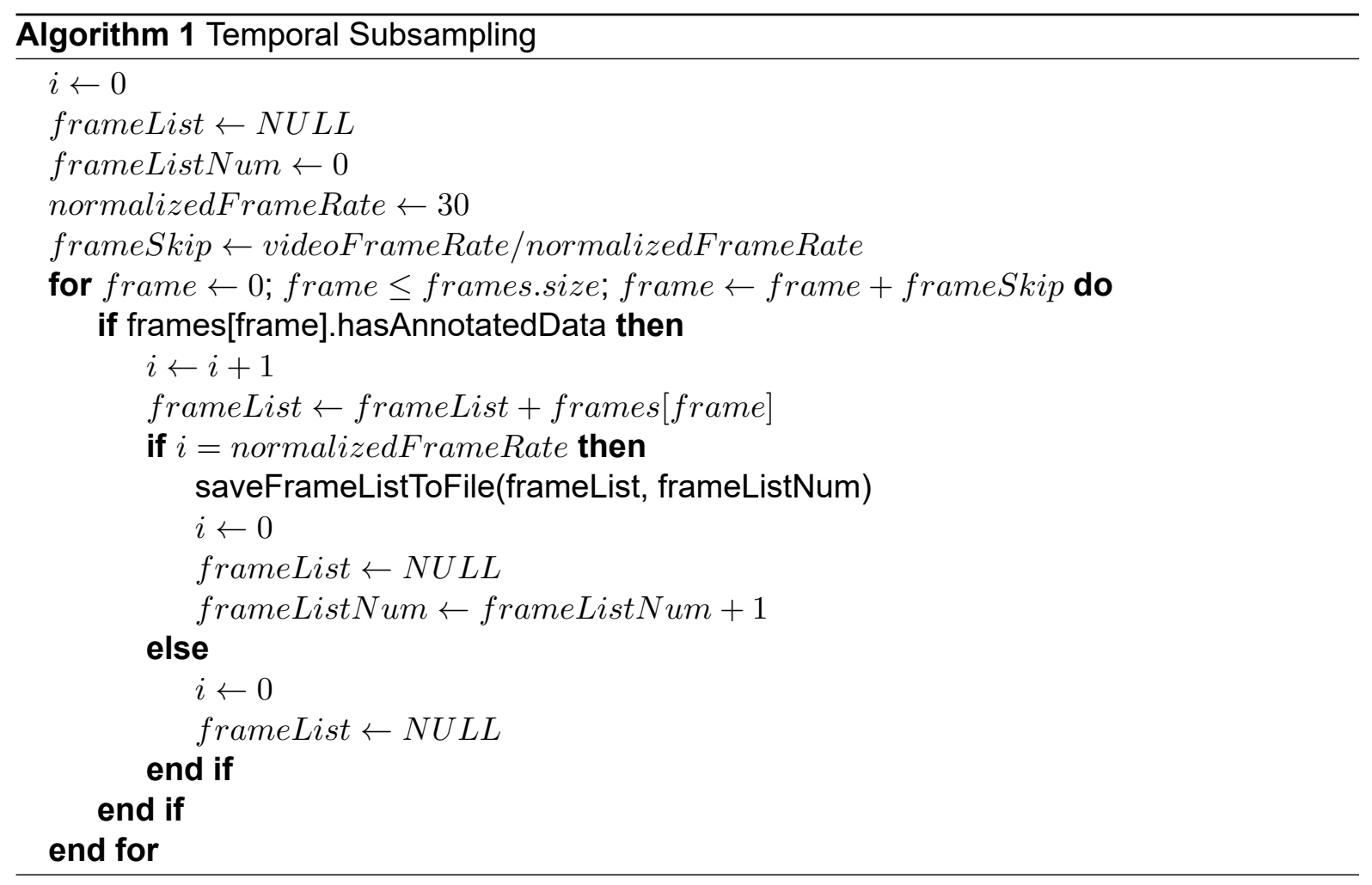

The increase counter value on the subsampling algorithm, was calculated based on the 
processed video's frame rate divided by the defined thirty frames per second rate. This enabled a consistent frame skipping.

Figure 5.2 exemplifies the motion data in signal form resulted from the feature extraction and subsampling process on two elements of the dataset.

\subsubsection{Discrete Fourier Transform (DFT)}

As the motion features were extracted, the result was signal data which needed to be analysed and broken into distinct features to be used by a statistical-based or distance-based classifiers.

The Fourier Transform is a powerful mechanism used in several fields from audio analysis [33], image compression [34] and in many fields of engineering. It's ability to transform any periodic function into frequency space provides for clear and distinguishable descriptors for signal data is the main reason for its widespread usage.

The Fourier Transform represents the infinite weighted sum of an infinite number of sinusoids (5.1), where $x$ represents the spacial domain, $\omega$ represents the frequency domain, $F(\omega)$ the frequency spectrum, $e^{i \theta}=\cos \theta+i \sin \theta$ and $i=\sqrt{-1}$.

$$
F(\omega)=\int_{-\infty}^{\infty} f(x) e^{-i 2 \pi \omega x} d x
$$

However, to compute the Fourier Transform in a discrete domain, the Discrete Fourier Transform (DFT) is used (5.2). A forward, one-dimensional DFT on an array $\mathrm{X}$ of $n$ complex numbers is the array $\mathrm{Y}$ given by where $0 \leq l<n$ and $\omega_{n}=\exp (-2 \pi \sqrt{-1} / n)$.:

$$
Y[k]=\sum_{j=0}^{n-1} X[j] \omega_{n}^{j k}
$$

The naïve implementation of this would mean an algorithm of $\Theta\left(n^{2}\right)$ complexity. To reduce the complexity and as such reduce computational costs, a Fast Fourier Transform (FFT) was used.

For this purpose, the design and implementation suggested by Frigo et. al. [35], in 2005, were used in the proposed Motion Classification method. This implementation computes any DFT for any length with a $O(n \log n)$ complexity, yielding significant performance gains.

The core of Frigo et. al's implementation basis is the use of Cooley-Tukey algorithm [36] and many of its variants [37]. But instead of focusing on a single best algorithm, Frigo et. al's approach is to instead build a planner which searches, using dynamic programming, several types of DFT implementation algorithms, called codelets, for the ones that would have optimal performance for the provided dataset, after breaking it down into simpler problems. The proposed implementation has over one hundred and fifty codelets, but more specific tuned ones can be produced with a special purpose compiler called genfft. 

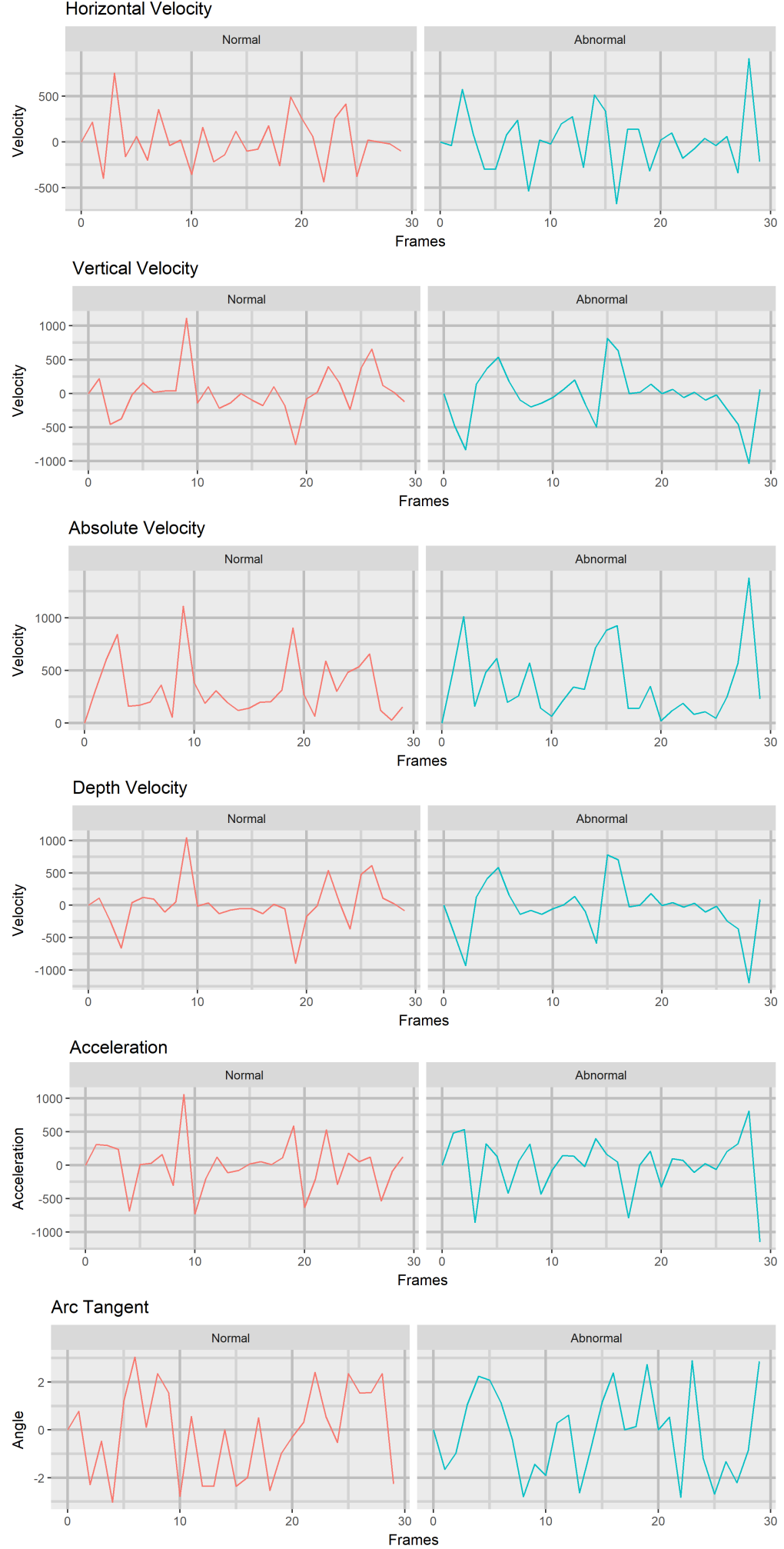

Figure 5.2: Extracted Motion Features 
These codelets are high level mathematical expressions of DFT algorithms and optimized for real data and machine specific instructions such as Single Instruction Multiple Data (SMID), which can be used to provide significant speed improvements on the computation of an FFT algorithm [38].

The planner is critical for the Frigo et. al's implementation performance, aiming to strike a balance between the planner's analysis time and the overall calculation time, using sensitive defaults, while enabling application specific parametrization.

While there are newer approaches that utilize more of multicore and GPU capabilities [39] yielding faster results, the performance of Frigo et. al's implementation was faster than the technical requirements of proposed Motion Classification method, and its proven reliability, demonstrated by the thousands of users and citations, proved to be a more trustworthy choice.

Ahmadi et. al. DWT approach was considered in place of the FFT, but it would introduce a higher mathematical complexity to the method and the FFT is very competitive with wavelets [40].

Figure 5.3 exemplifies the motion data in frequency space resulted from the FFT over the feature extraction data on two elements of the dataset.

\subsubsection{Classification}

Once the motion features were extracted and processed, two types of classification approaches were tested: statistical based and distance based. Statistical based are the more traditional data mining techniques, while distance based are more specific for comparing signals / shapes. The objective is to compare the produced results and ascertain the ones that obtained the best results, while validating if the extracted features were adequate for the pathology classification.

\section{Statistical Based Approaches}

Three types of statistical based classifiers were tested:

- C5.0 - A Decision Tree algorithm

- SVMs

- Random Forest - An ensemble type of algorithm

They were chosen as each represents a different type of approach towards statistical analysis, and as such, were expected to produce different results.

The C5.0 decision tree algorithm is the successor of the C4.5 algorithm by Quinlan [41]. It has faster compute time, higher accuracy, lower memory footprint as it creates smaller trees and has lower error in unseen cases [42] than its predecessor. 

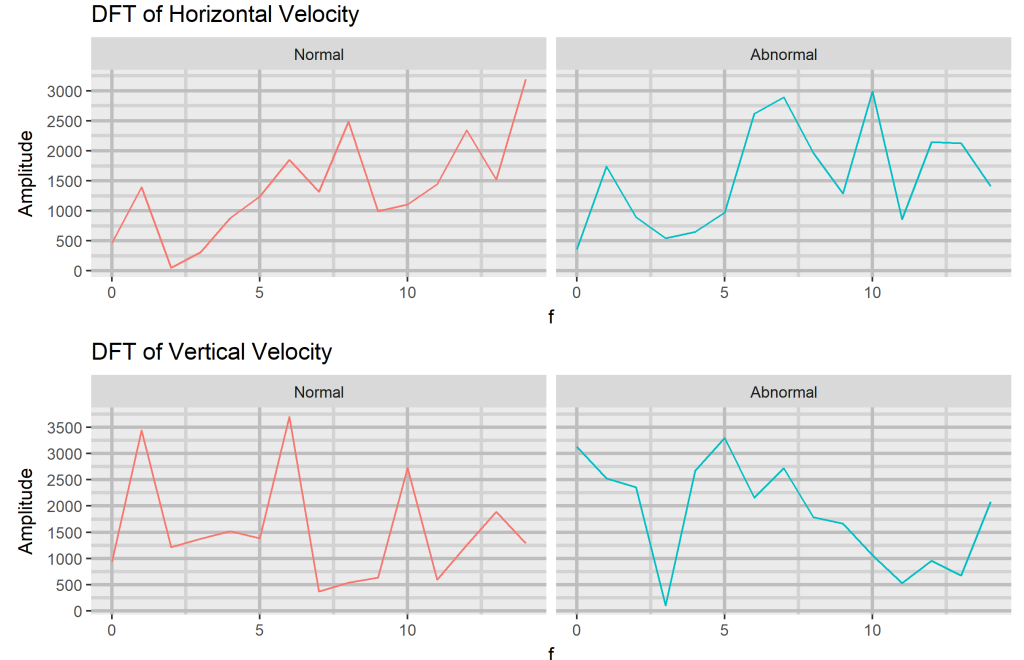

DFT of Absolute Velocity
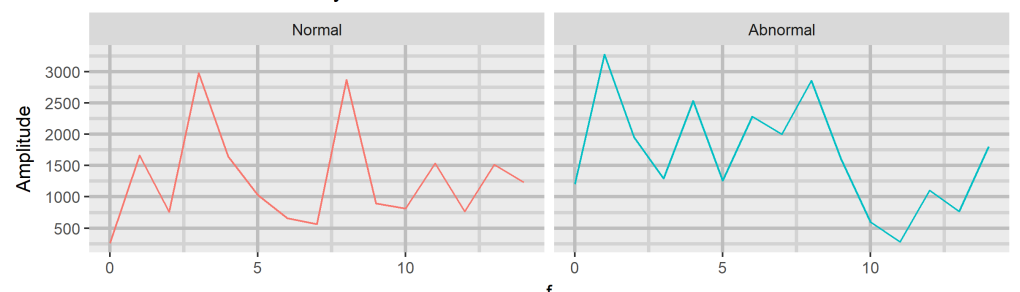

\section{DFT of Depth Velocity}
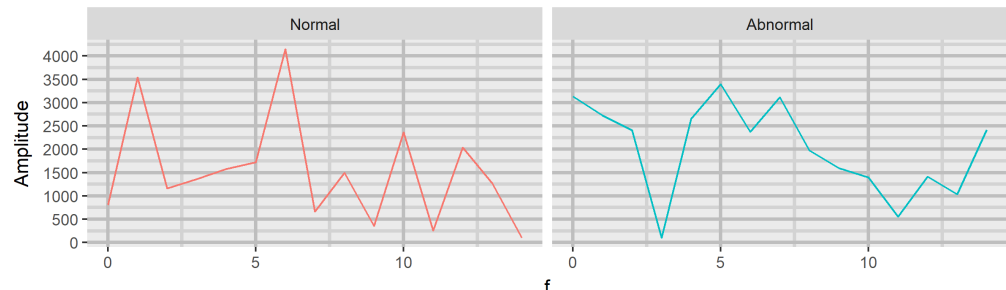

f

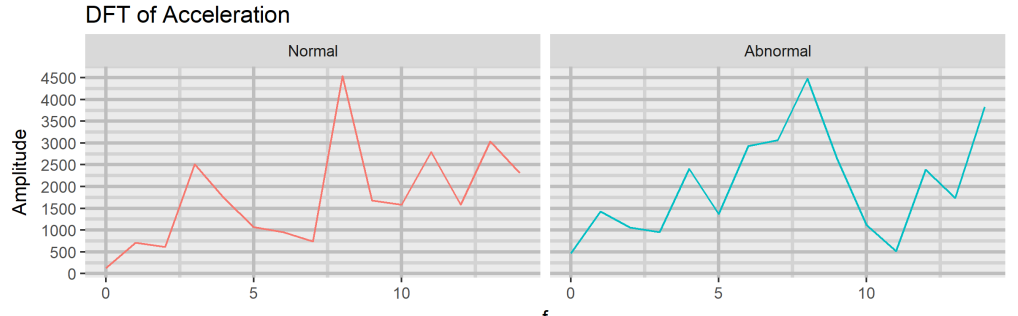

DFT of Arc Tangent

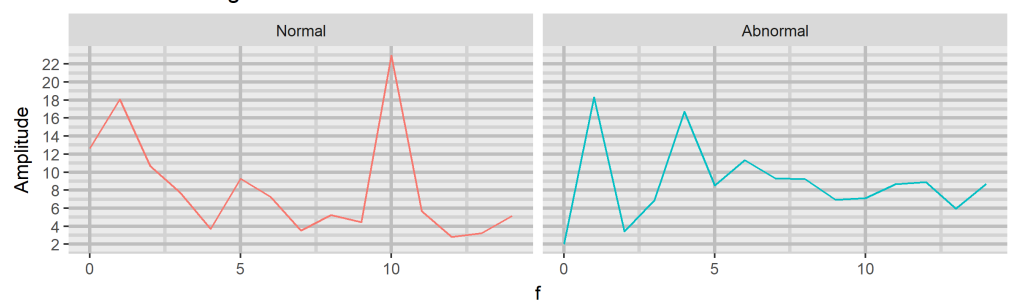

Figure 5.3: DFT of Motion Features 
Th C5.0 algorithm uses a statistical approach to create rule-based models. Decision trees operate by deciding if a branch should be split based on the normalized gain ratio that would occur from this operation, this based on the attributes being analysed. The C5.0 algorithm uses information gain as the gain criterion, rather than the Gini Index used by the CART algorithm [43].

The information gain is based on calculating the entropy measure (5.3) of the dataset's attributes (5.4).

$$
\begin{gathered}
\text { Entropy }=-\sum_{i=1}^{n} p_{i} \log _{2}\left(p_{i}\right) \\
\text { InformationGain }=\operatorname{Entropy}(\text { Target })-\operatorname{Entropy}(\text { Attribute })
\end{gathered}
$$

C5.0 adds to that with boosting, based on the work by Freund Et. Al. [44], configurable misclassification costs, support for more data types and it can also automatically winnow the attributes [45], discarding the ones that are not relevant, making it more adequate for datasets with high dimensionality. It also does pruning of the tree, reducing its size and it also serves to mitigate overfitting of the decision tree model.

As seen on Figure 5.4 which demonstrates the resulting C5.0 tree calculated from the training dataset, decision trees are inherently intuitive, making their results more easily understood by non-technical users.

SVMs have been successfully used for face detection [46], bio-informatics [47], text-mining [48] and many other classification tasks. The core of the SVM algorithm is mapping the dataset into a new space of higher dimension and then find the linear decision surface, aka hyperplane, that can separate the classes. Figure 5.5 ilustrates this process.

As there can be an infinite number of hyperplanes separating two classes, SVMs use an approach called Maximum Margin Hyperplace, which uses quadratic programming to optimize the solution. They are solved efficiently by using greedy algorithms in order to obtain the hyperplane with the highest distance between border points, as to avoid overfitting the SVM results.

However, to solve the optimization equation, dot products are required. For high dimensional spaces they can be very computationally demanding. To solve this issue a so called "kernel trick" is used. These are functions that utilize the fact that $K(x, z)=\phi(X) \cdot \phi(Z)$ to utilize more efficient functions instead of the costly dot products, although these functions must satisfy Mercer conditions [50] to be able to be utilized. There are multiple kernels available, such as the Linear kernel, the Gaussian kernel, the Exponential kernel, the Polynomial kernel, among many others.

Random Forests, as proposed by Breiman [51] in 2001, is an ensemble algorithm, so it's a collection of models used together to produce predictions. Ensemble algorithms tend to produce good results based on these three fundamental reasons [52]:

- Statistical 


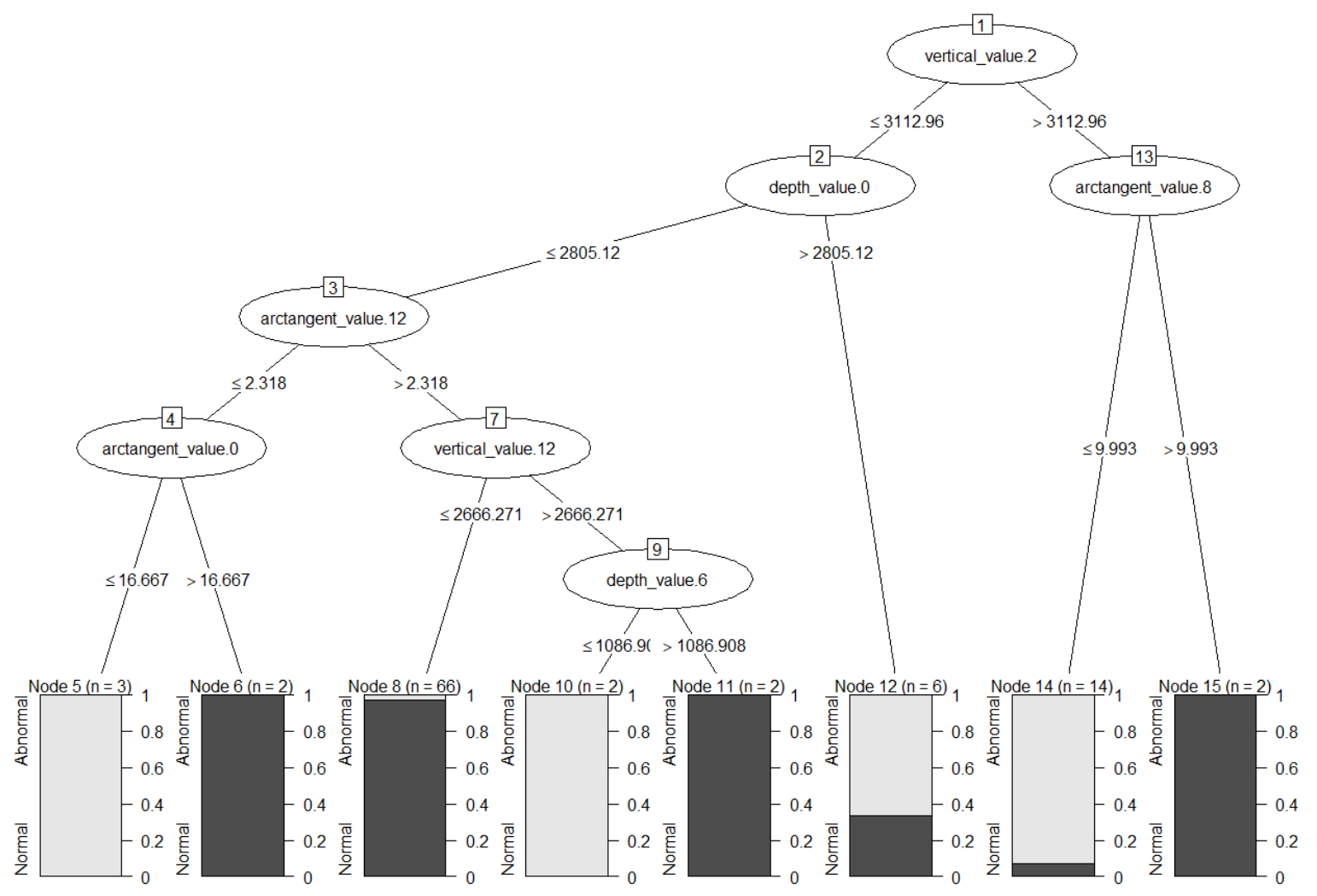

Figure 5.4: C5.0 Resulting Decision Tree
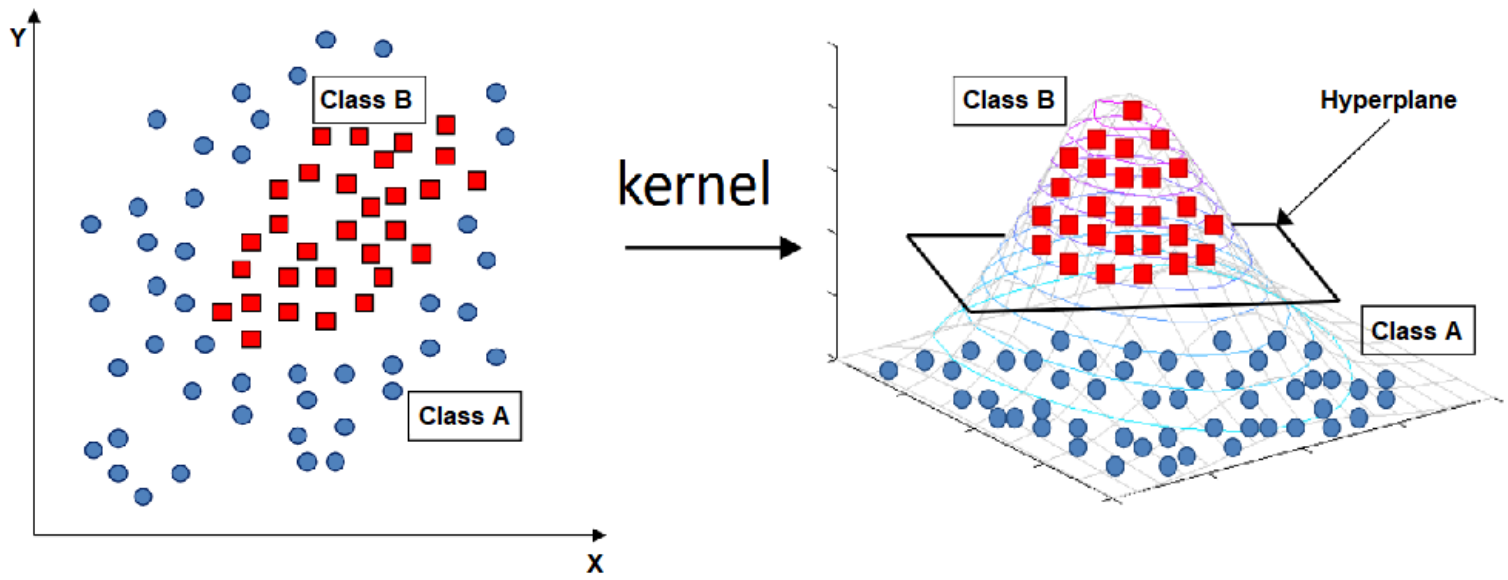

Figure 5.5: Support Vector Machine (SVM) - Adapted from [49]

As learning algorithms try to identify the best hypothesis in space $H$, multiple hypothesis can have the same accuracy, especially if the training data is significantly smaller than the space $H$, which can lead to a selection of an hypothesis that while good on the training data, is inadequate on the test data. As the ensemble averages these hypoth- 
esis votes, it reduces the risk of using a wrong classifier. Figure 5.6(A) exemplifies this, as the point $f$ represents the true hypothesis function, the $h 1-h 5$ points represent the ensemble hypothesis. By averaging these hypotheses, a more accurate approximation of the $f$ hypothesis can be found.

- Computational

As optimal training for some classification algorithms can be NP-Complete [53] [54], the usage of heuristics requires a larger dataset to build the statistical problem. Ensembles can be constructed by using random starting points an doing a local search on them, providing a potentially better approximation, at a lower computational cost. Figure 5.6(B) illustrates this concept.

- Representational

As the true hypothesis function $f$ maybe outside the space $H$, as Figure 5.6(C) exemplifies, the weighted sum of hypothesis generated by the ensemble algorithm can expand this space of possible hypothesis, making it more suitable for classifying unseen data.

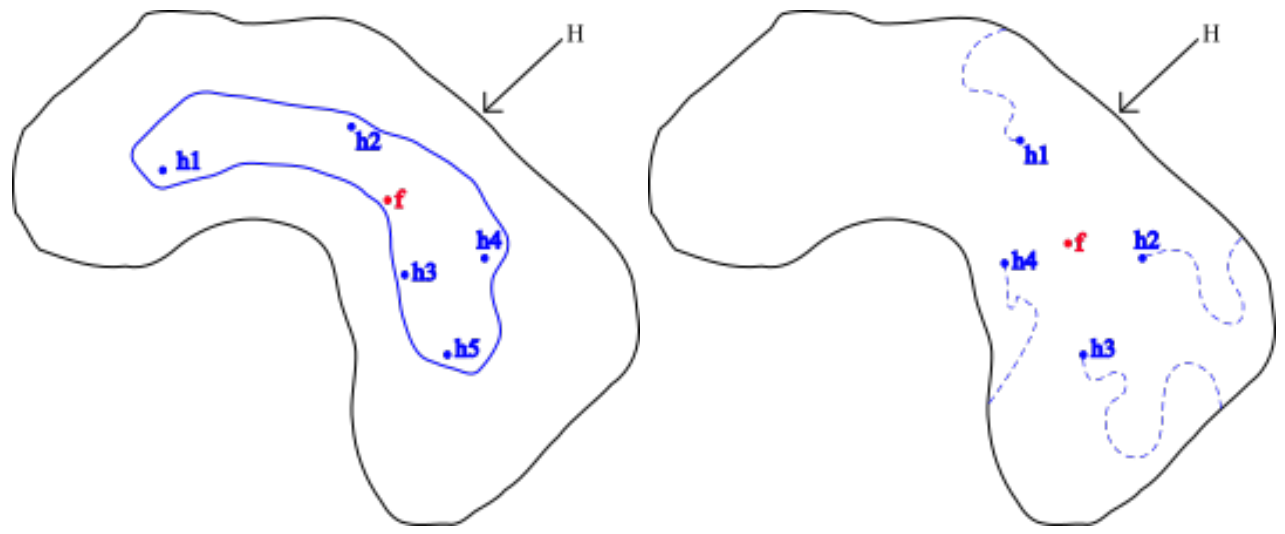

(A) Statistical

(B) Computational

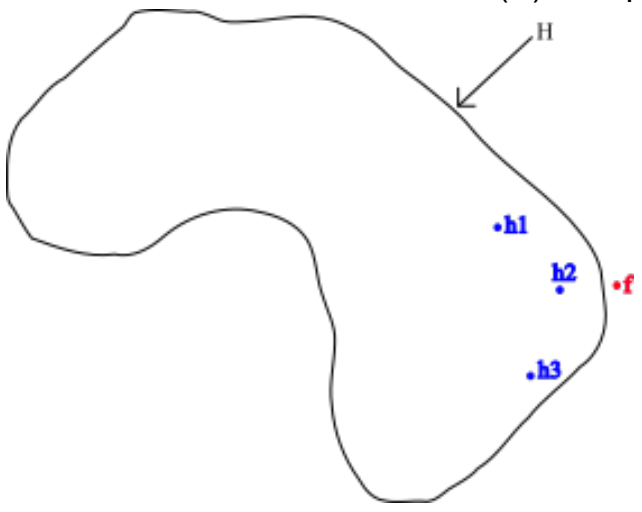

(C) Representational

Figure 5.6: Ensemble's Fundamental Reasons for Producing Good Results (Based on [52]) 
Random Forests utilizes the concepts of bagging predictors [55] and also the use of random predictors. Bagging predictors is a method for creating $n$ number of models based on samples created with replacement of the same size as the training dataset, ensuring diversity between the $n$ models as they are to be used alongside a very sensitive base learner. Random predictors are a variation of the bagging predictor but uses samples of variables instead of samples of the full dataset.

The Random Forests algorithm executes the following steps, while in a loop for each $n$ model:

- Create a sample with replacement of the same size as the training dataset

- Creates a classification (or regression) tree - The nodes however are chosen at random from a subset of predictors

\section{Distance Based Approaches}

Two types of distance based algorithms were tested:

- Dynamic Time Warping (DTW)

- Hausdorff's Distance

Dynamic Time Warping (DTW) has been used in multiple areas, ranging from biometrics, handwriting and signature matching, time series clustering and electro-cardiogram analysis [56] [57]. The DTW algorithm aims to find an optimal match between two time series by using local compression and stretching, in order to make then resemble each other as much as possible and then calculate the distance.

The algorithm uses a non-negative local distance function, which is used to build a distance matrix between all the pairwise distances of the time series. After this step, the DTW algorithm finds the alignment path thought the cost matrix, which passes through the lowest cost areas. To find the optimal warping path, dynamic programming is used in order to break down the problem and archive an $O(N M)$ complexity. Figure 5.7 exhibits the alignment process and result.

This dynamic warping makes it well suited to compare signal data, such as the DFT data extracted from the AML's motion. This is done by stablishing a baseline of normal DFT data and Pathological DFT data, a procedure done by calculating the mean value for each data point. Afterwards the comparison is done on each element of the dataset to these baselines, doing the classification by which baseline is the closest one.

Hausdorff's Distance has been used for comparing images [58], shape matching [59], and even face detection [60]. It is denoted as $h(P, Q)=\max _{p \in P} \min _{q \in Q}\|p-q\|$, which means that it finds the $p$ that is the furthest from $Q$ and obtains it's distance from its nearest $q$ point. Figure 5.8 demonstrates this operation. 


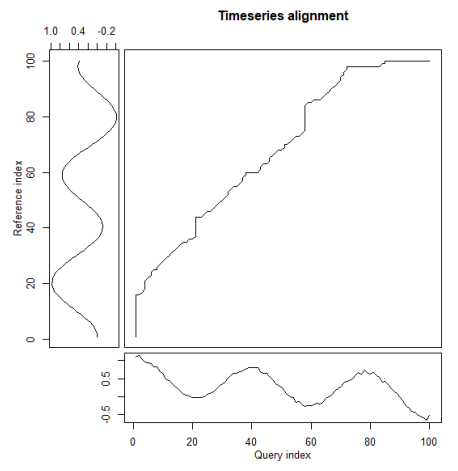

(A) DTW Alignment

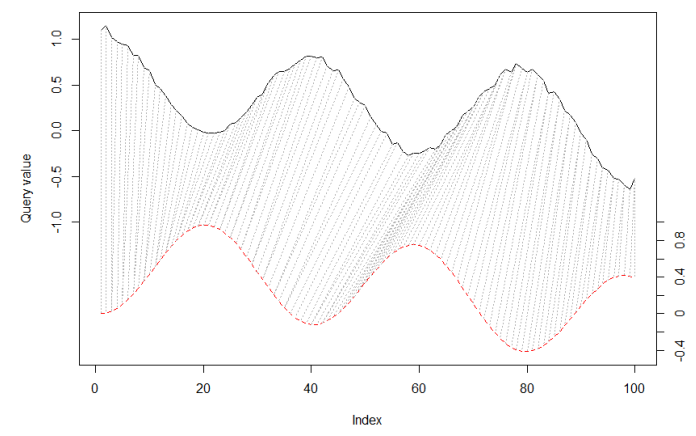

(B) ]

Figure 5.7: Dynamic Time Warping (DTW)

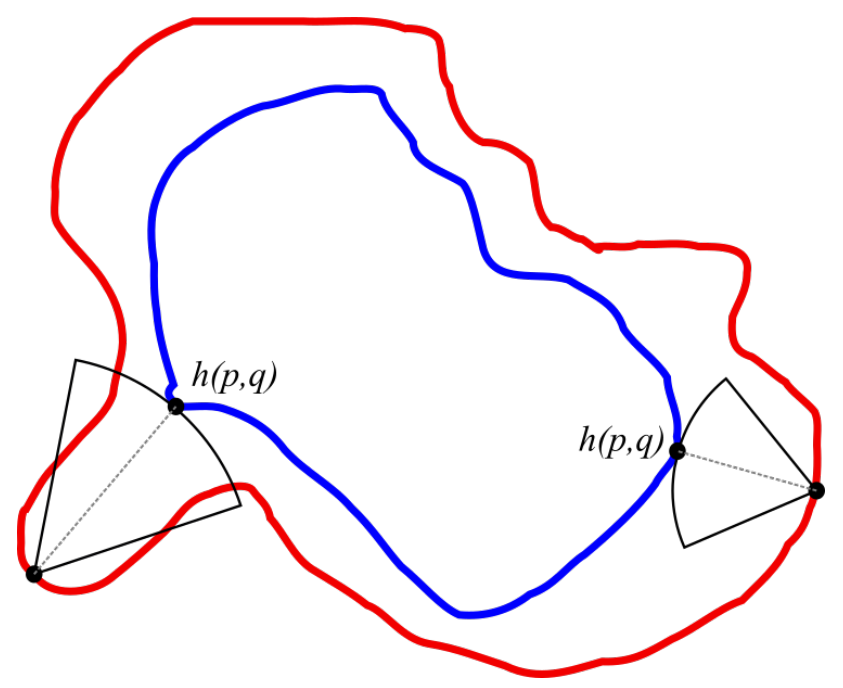

Figure 5.8: Hausdorff's Distance

For two-dimensional data Hausdorff's Distance can be optimized by use of a Voronoi diagram to archive a $O(n \log n)$ complexity. There are also variations that utilize the average of the points distances, making it more less sensitive to outliers.

While the Hausdorff's Distance base algorithm is simple, it's well suited to use as a dissimilarity metric between visual elements as it's slightly insensitive to small variations and serves to compare the overall shape of the images. While the DFT data is not an image per se, the signal data does produce a form of shape, reason why this algorithm was chosen. To perform the classification on the dataset, the same procedure of stablishing a baseline and comparison as used on the DTW was performed. 


\subsection{Results}

For the evaluation of the statistical algorithms, a Leave One Out Cross Validation method was utilized due to the dataset's small size. The distance algorithms were evaluated based on a built Confusion Matrix using the method outlined above for the classification. This was done for each of the features extracted from the dataset. These results are outputted on Tables 5.1(A), 5.1(B), 5.1(C), 5.1(D), 5.1(E) and 5.1(F). Figure 5.9 does a graphical comparison of these results.

\begin{tabular}{|c|c|c|c|c|c|c|c|c|c|c|c|}
\hline & DTW & Hausdorff & C5.0 & SVM & RandomForest & & DTW & Hausdorff & C5.0 & SVM & RandomForest \\
\hline Accuracy & 0.52 & 0.67 & 0.75 & 0.75 & 0.74 & Accuracy & 0.71 & 0.26 & 0.80 & 0.80 & 0.81 \\
\hline Precision & 0.79 & 0.21 & & & 0.33 & Precision & 0.58 & 0.96 & 0.60 & 0.73 & 0.71 \\
\hline Recall & 0.31 & 0.28 & 0.00 & 0.00 & 0.04 & Recall & 0.44 & 0.24 & 0.62 & 0.33 & 0.42 \\
\hline$F 1$ & 0.45 & 0.24 & & & 0.07 & $F 1$ & 0.50 & 0.39 & 0.61 & 0.46 & 0.53 \\
\hline Neg Pred Value & 0.42 & 0.82 & 1.00 & 1.00 & 0.96 & Neg Pred Value & 0.75 & 0.03 & 0.38 & 0.67 & 0.58 \\
\hline
\end{tabular}

\begin{tabular}{|c|c|c|c|c|c|c|c|c|c|c|c|}
\hline \multicolumn{6}{|c|}{ (A) Horizontal Velocity } & \multicolumn{6}{|c|}{ (B) Vertical Velocity } \\
\hline & DTW & Hausdorff & C5.0 & SVM & RandomForest & & DTW & Hausdorff & C5.0 & SVM & RandomForest \\
\hline Accuracy & 0.61 & 0.31 & 0.65 & 0.74 & 0.71 & Accuracy & 0.75 & 0.27 & 0.77 & 0.78 & 0.80 \\
\hline Precision & 0.46 & 0.96 & 0.00 & 0.00 & 0.00 & Precision & 0.50 & 1.00 & 0.55 & 0.62 & 0.69 \\
\hline Recall & 0.31 & 0.26 & 0.00 & 0.00 & 0.00 & Recall & 0.50 & 0.25 & 0.50 & 0.33 & 0.38 \\
\hline$F 1$ & 0.37 & 0.41 & & & & $F 1$ & 0.50 & 0.40 & 0.52 & 0.43 & 0.49 \\
\hline Neg Pred Value & 0.66 & 0.10 & 1.00 & 1.00 & 1.00 & Neg Pred Value & 0.84 & 0.03 & 0.50 & 0.67 & 0.62 \\
\hline \multicolumn{6}{|c|}{ (C) Absolute Velocity } & \multicolumn{6}{|c|}{ (D) Depth Velocity } \\
\hline & DTW & Hausdorff & C5.0 & SVM & RandomForest & & DTW & Hausdorff & C5.0 & SVM & RandomForest \\
\hline Accuracy & 0.51 & 0.33 & 0.71 & 0.75 & 0.71 & Accuracy & 0.60 & 0.71 & 0.71 & 0.75 & 0.73 \\
\hline Precision & 0.62 & 0.92 & 0.00 & & 0.00 & Precision & 0.54 & 0.00 & 0.00 & & 0.00 \\
\hline Recall & 0.28 & 0.26 & 0.00 & 0.00 & 0.00 & Recall & 0.32 & 0.00 & 0.00 & 0.00 & 0.00 \\
\hline$F 1$ & 0.38 & 0.40 & & & & $F 1$ & 0.40 & & & & \\
\hline Neg Pred Value & 0.47 & 0.14 & 1.00 & 1.00 & 1.00 & Neg Pred Value & 0.62 & 0.95 & 1.00 & 1.00 & 1.00 \\
\hline
\end{tabular}

(E) Acceleration

(F) Arc Tangent

Table 5.1: Evaluation Metrics for each Classifier by Features

These initial results show that Statistical Based Classifiers, as seen in 5.1 and 5.9(B), had a slightly higher accuracy and higher negative prediction value, meaning less pathological cases were classified as non pathological - a false negative, but much lower precision and recall values then the Distance Based Classifiers. The DTW had the best median performance, with a mean accuracy of $61.51 \%$, mean precision of $58.33 \%$, mean recall of $35.82 \%$, mean $\mathrm{F} 1$ of $43.31 \%$ and a mean negative predicated value of $62.56 \%$. SVM, had low values for precision, Recall and $\mathrm{F} 1$ but the highest mean negative predicated value of $88.89 \%$.

The analysis by features, as seen in 5.1 and $5.9(\mathrm{~A})$, indicated that vertical velocity yielded the best overall results, with a mean accuracy of $67.84 \%$, mean precision of $71.66 \%$, mean recall of $41.14 \%$, mean $\mathrm{F} 1$ of $49.71 \%$ and a mean negative predicated value of $48.12 \%$. Depth velocity yielded very similar results, but with a slightly better mean negative predicated value of $53.09 \%$. Arc tangent had the highest mean negative predicated value at $91.23 \%$, but overall poor results.

This initial classification results were based on a methodology that only used one set of features per training / testing. Based on the analysis of these results, the two features that yielded the best overall results, vertical velocity and depth velocity, and the feature that had the 


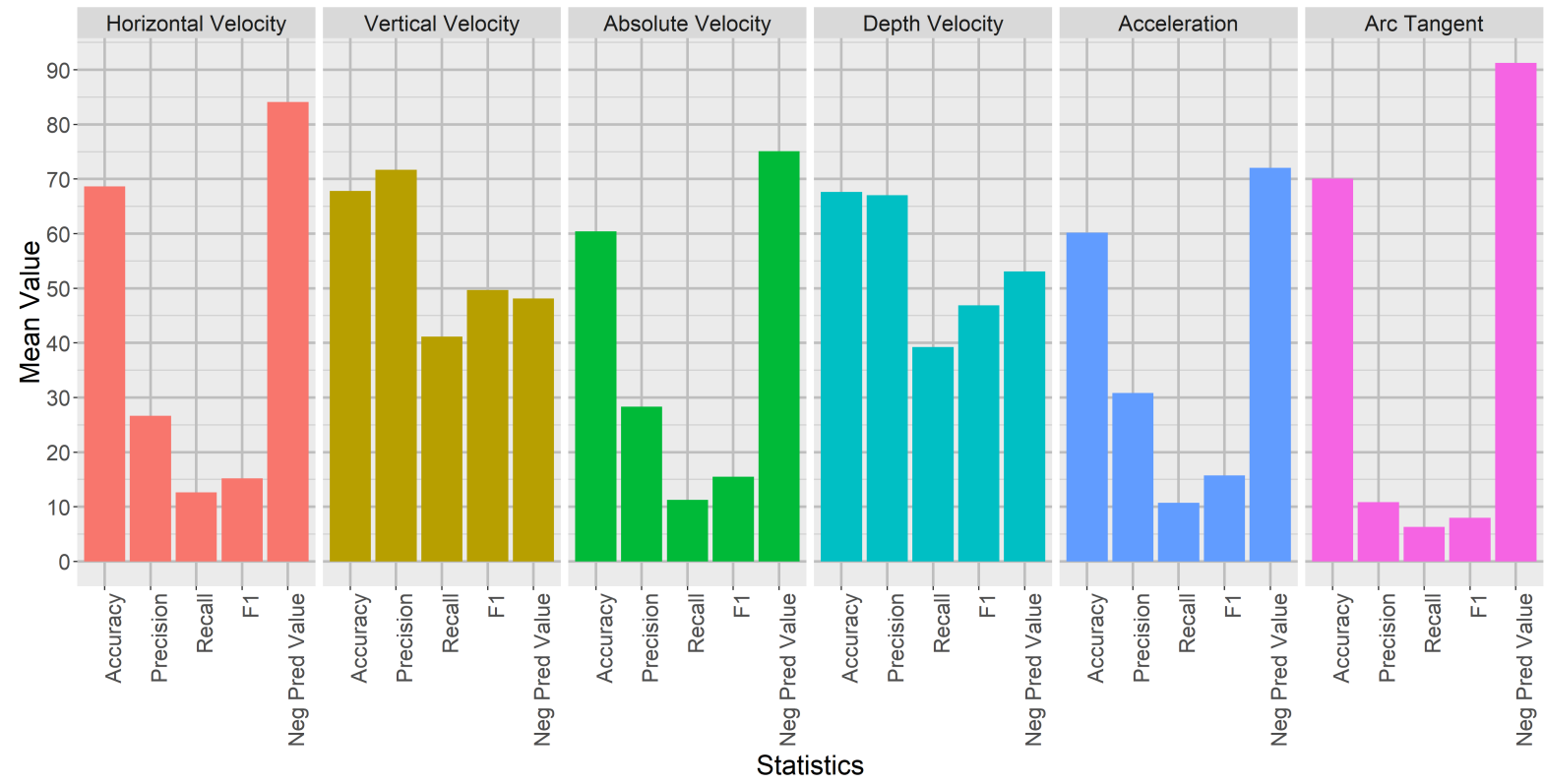

(A) Mean Values of the classifiers evaluation values by Feature

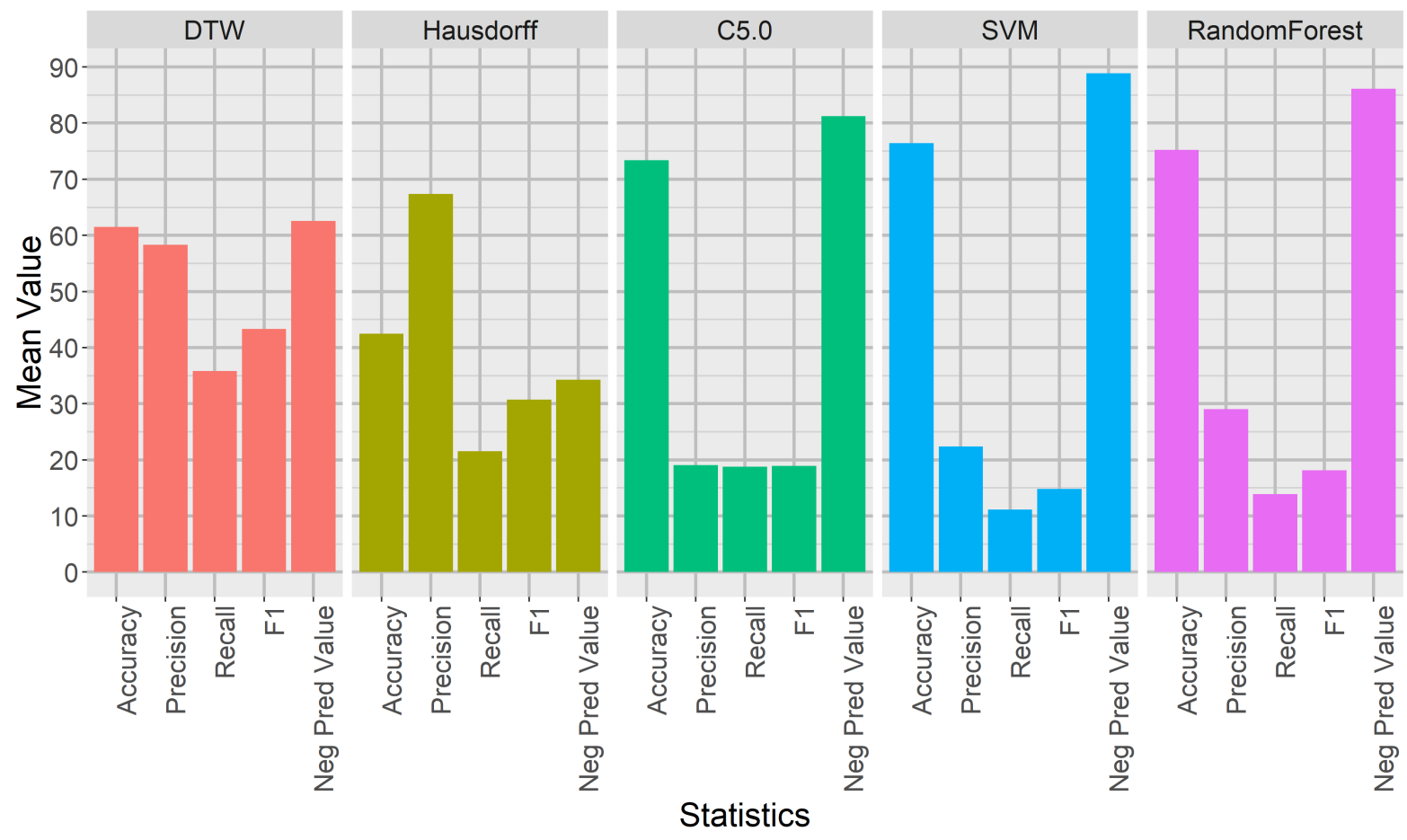

(B) Mean Values of the features evaluation values by Classifiers

Figure 5.9: Evaluation Metrics for each Classifier by Features - Graphical Comparison

highest mean negative predicated value, arc tangent, were used in combination to potentially improve the overall classification results.

As the dataset was imbalanced, the usage of cost-sensitive learning [61] was also explored, aiming to reduce the False Negatives, as it's a significant metric in this case. However as the 
initial results didn't yield relevant improvements, and in some cases even causing lower scores, this approached was not utilized in these classification results.

For the statistical based classifiers, these features where placed together in a joint dataset, and left to the respective algorithms to extract the most relevant fields for classification, while the distance based classifiers utilized a voting algorithm developed to extract the final classification result.

This voting algorithm run a loop with the distance based algorithms for each feature, registering each of the outputted classification results as a single vote. After this procedure, the final classification was defined based on the most voted classification result.

The results of this combined feature set classification are on Table 5.2. Figure 5.10 exhibits the graphical comparison between the different classifiers results

\begin{tabular}{l|rrrrr} 
& DTW & Hausdorff & C5.0 & SVM & RandomForest \\
\hline Accuracy & 0.22 & 0.73 & 0.82 & 0.76 & 0.81 \\
Precision & 0.42 & 0.04 & 0.64 & 0.56 & 0.71 \\
Recall & 0.14 & 0.25 & 0.67 & 0.21 & 0.42 \\
F1 & 0.21 & 0.07 & 0.65 & 0.30 & 0.53 \\
Neg Pred Value & 0.15 & 0.96 & 0.33 & 0.79 & 0.58 \\
\hline
\end{tabular}

Table 5.2: Combined Features Results

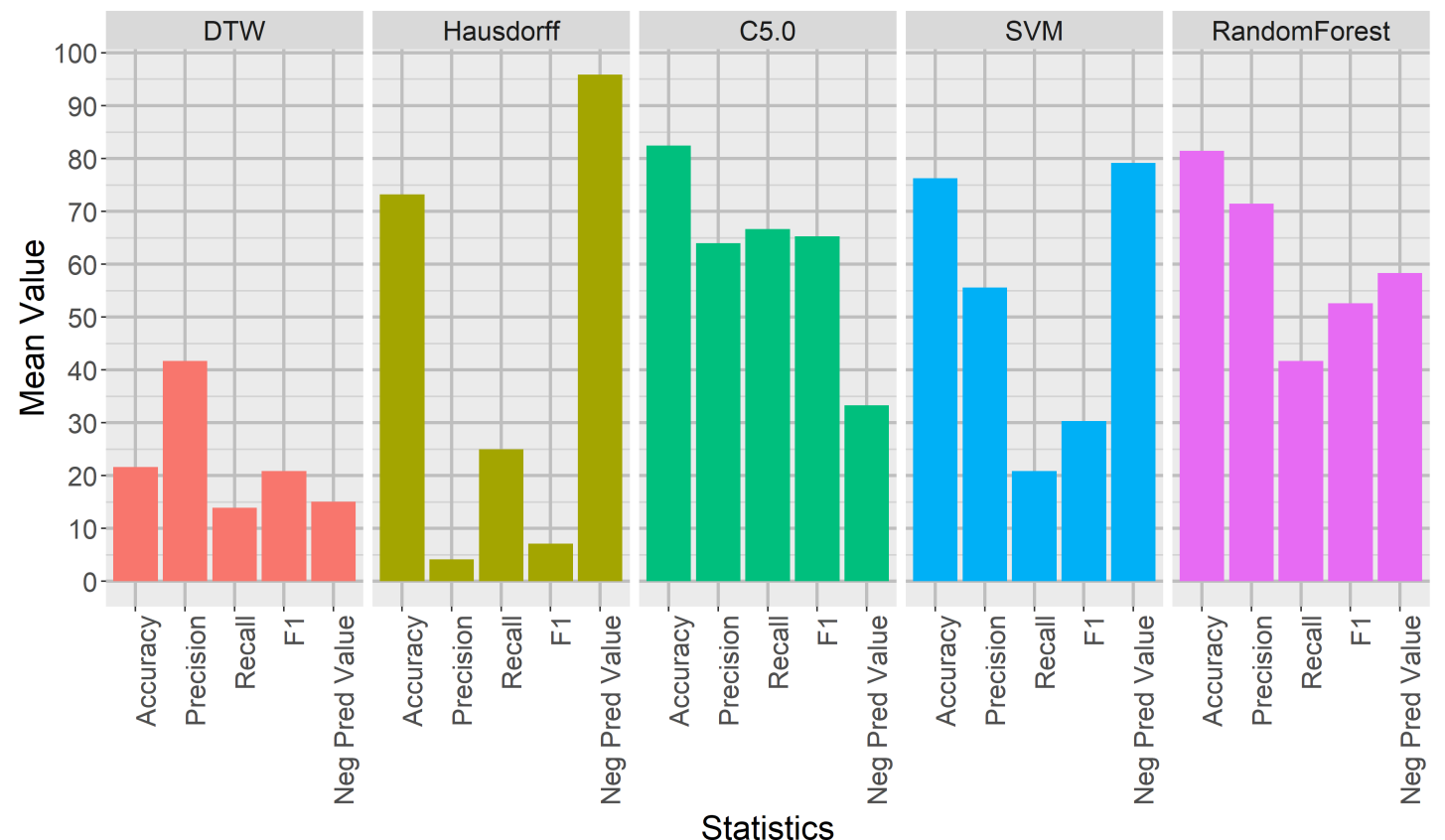

Figure 5.10: Combined Features Results - Graphical Comparison

The overall accuracy, precision, recall and F1 metrics, as seen in 5.2 and 5.10, was improved on the statistical based classifiers, with the used of the three features in a combined feature set, however Random Forest and C5.0 had a high decrease (28\%-48\%) in negative 
predicated value metric, while the SVM had a smaller decrease $(10 \%)$. The DTW had and overall decrease, while the Hausdorff saw an increase on accuracy (31\%) and a very high increase negative predicated value $(62 \%)$, while precision, recall and $F 1$ saw decreases. No classifier presented a good overall result. The high accuracy and poor precision and recall results on the DTW indicate that this classifier was not being able to classify cases as pathological.

\subsection{Discussion}

The C5.0 using the combined features produced better than average results in terms of accuracy (82\%), precision (64\%), recall (67\%) and F1 (65\%), but very poor results for negative predicated value (33\%), a critical metric in this case. Hausdorff had very high results $(96 \%)$ on this metric. Figure 5.4 exhibits the $\mathrm{C} 5.0$ resulting tree.

These results could be improved by using techniques such as Principal Components Analysis (PCA), SVM kernel tuning, algorithm combination, among others. However, the initial goal was to test if the proposed method extracted features were discriminative enough to be utilized for classification.

The small dataset presented limitations, especially as the pathological data was a very small and imbalanced sample, presenting which led to poorer results for the statistical classifiers in terms of accuracy, precision and recall, especially on the initial testing, as it failed to better discriminate between normal $x$ pathological cases. Additional techniques for improving classification in this situation, such as generating synthetic samples in order to reduce the imbalance of the dataset, such as Synthetic Minority Over-Sampling Technique (SMOTE) were analysed but considered inadequate, as the synthetic data might not be representative of the medical condition.

Using the Shapiro-Wilk normality test, it was observed that on the extracted features, very few frames had a normal distribution. This is probably due to the fact that the cycles in the features where not align, as the dataset had no annotated data with this information. Aligning these cycles would lead to a better statistical analysis of each cycle point, enabling the observation of normal/abnormal behaviour thresholds in a simpler manner, and possibility lead to better classification results from the Statistical based and specially Distance based classifiers, which suffered from poor results on the combined dataset.

The current results indicate that the presented method requires further improvements in order to provide a adequate aid for physicians on RHD screening. 


\section{Chapter 6}

\section{Conclusion}

\subsection{Discussion}

In this thesis, RHD and its impact were described, as well as the ultrasound technology used to screen it. State of the art methods for thickness analysis and motion classification were reviewed and analysed.

To further aid in the analysis and screening of RHD a methodology for objectively measuring the thickness profile of the anterior mitral valve leaflet, with well-defined landmarks was proposed. The analysis done from the extracted data using the proposed methodology indicated that the measurement of thickness is most adequate at the tip of the AML and when the valve is open or straight, as it was observed a statistical and median difference of the thickness between normal and pathological cases. It also provided information of the overall thickness variance along the leaflet's shape and pathology. This statistical information could also be of use as shape descriptors for automatic segmentation algorithms.

The proposed framework can also be used to validate result produced by automatic segmentation algorithms, as seen on 6.1. These results indicate that Sultan et al.'s approach yielded similar results to the manual annotation on the Tip, but presented thicker results at the Mid -1, Mid -2 and Tail quartiles. These results were obtained by using the framework on the automatic segmentation results on the same dataset.

The defined control points enable a reduction in the inter and intra-observer variability of thickness measurements, as it makes the thickness measurements directly comparable, as the methodology produces a constant result on the same image. This feature can also be of use for the automatic validation of segmentation algorithms, as it makes the critical points of the leaflet's overall shape comparable with the expert operator's segmented area. The proposed method was also very computationally efficient, enabling real-time usage.

It was also proposed a motion classification methodology to detect pathological motion patterns. It built on the thickness measurements methodology, and proposed motion features extractions methods. The end result was a decision tree with $82 \%$ accuracy, $64 \%$ precision, 


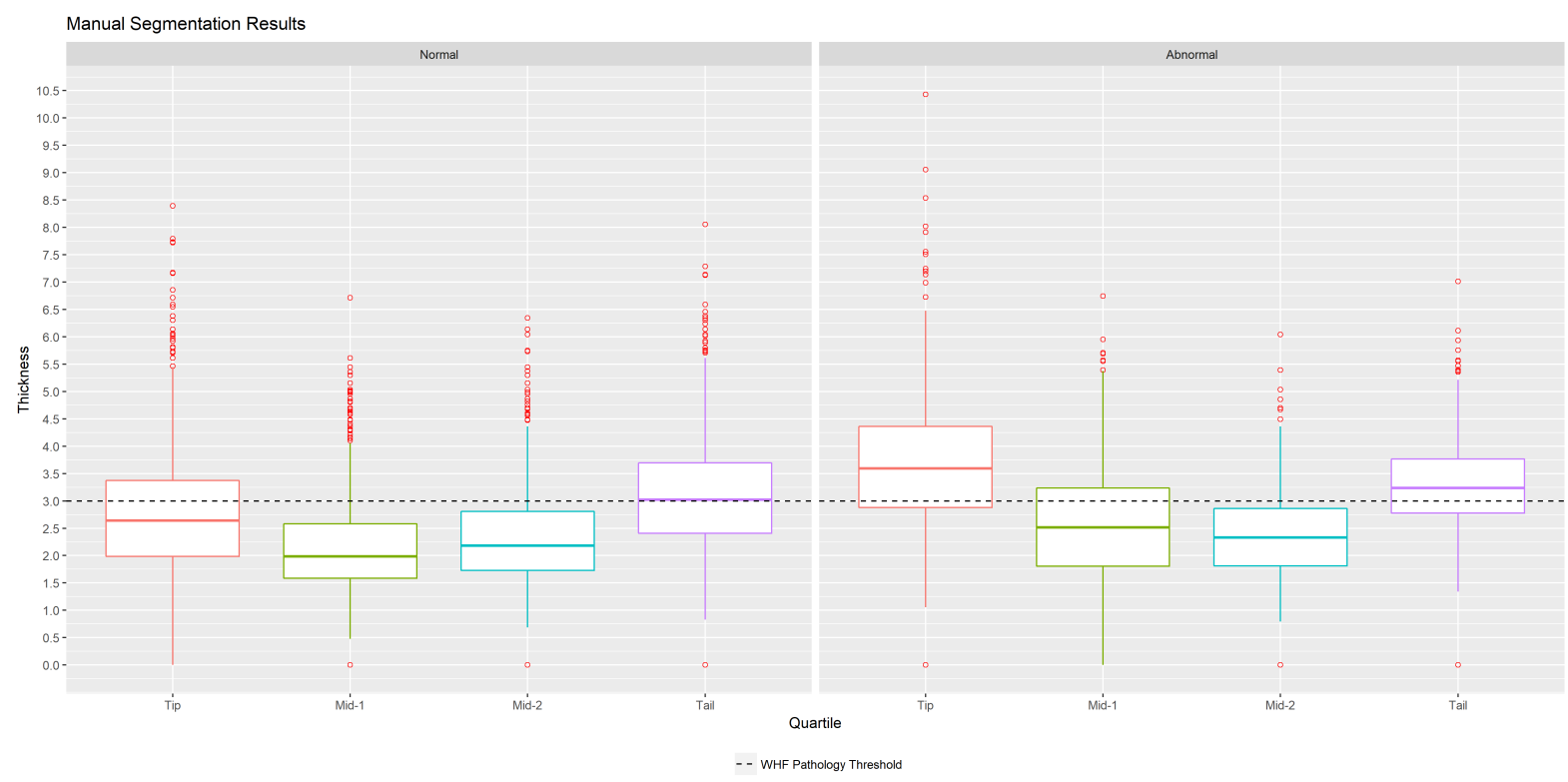

(A) Manual Segmentation Thickness Results

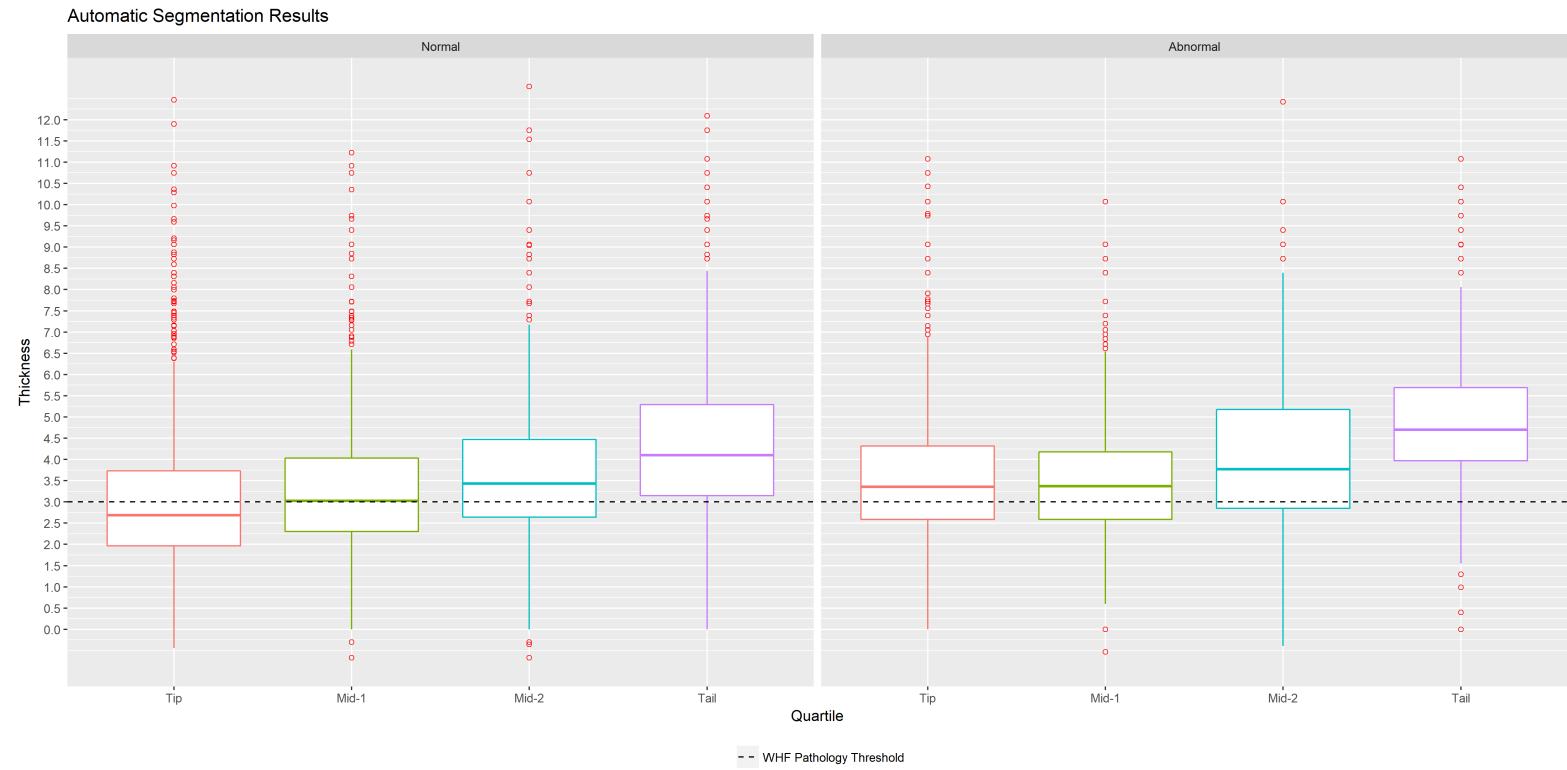

(B) Automatic Segmentation Thickness Results - Sultan et. al.'s Approach [62]

Figure 6.1: Manual x Automatic Segmentation Comparison using the Thickness Framework

$67 \%$ recall $65 \% \mathrm{~F} 1$ and $33 \%$ negative predicated value and a Hausdorff classification with $96 \%$ negative predicated value, which could be potentially used together in future work. It also had a low computational cost.

The small dataset, the AML's irregular shape and motion and insufficient annotated data presented a challenging problem for both methodologies. The Thickness Profile Extraction was able to produce consistent results and multiple statistical data on the AML's shape in different positions and pathologies. The Motion Classification established a ground work for feature 
extraction of the AML's motion, which can be further developed in future work.

\subsection{Future Work}

Future work on the Thickness Profile Extraction can improve the approach to make it more comparable to existing annotation by doing a few measurements per quartile, shifting a bit the position from the midpoint and calculating the mean between these measurements. This would likely produce closer results to a human arbitrary measuring location. The implementation can also be further optimized to reduce computational costs with parallelization and memory optimizations.

The paper by Sultan et al. [62] proposed a method called Virtual M-Mode that could be potentially used alongside with Dhutia et al. [15] method to obtain the cardiac cycle information. This could enable the syncing of the extract motion features from the Motion Classification methodology, with could lead to well defined distributions, that could then be analysed as the thickness data was and also produce better classification results. It could also negate the need for the DFT and reduce computational costs.

Other approaches could be the use of DWT in place of the DFT, as Ahmadi et. al [17] proposed. Separation of the pathological group into more groups, such as excessive motion and restricted motion could also potentially improve results, as the motion patterns would be more distinct. 


\section{Bibliography}

[1] J. C. Lu, C. Sable, G. J. Ensing, C. Webb, J. Scheel, T. Aliku, P. Lwabi, J. Godown, and A. Beaton, "Simplified rheumatic heart disease screening criteria for handheld echocardiography," J Am Soc Echocardiogr, vol. 28, no. 4, pp. 463-9, $2015 . \quad$ Lu, Jimmy C Sable, Craig Ensing, Gregory J Webb, Catherine Scheel, Janet Aliku, Twalib Lwabi, Peter Godown, Justin Beaton, Andrea eng KL2TR000076/TR/NCATS NIH HHS/ UL1TR000075/TR/NCATS NIH HHS/ Multicenter Study Research Support, N.I.H., Extramural Research Support, Non-U.S. Gov't J Am Soc Echocardiogr. 2015 Apr;28(4):463-9. doi: 10.1016/j.echo.2015.01.001. Epub 2015 Feb 7.

[2] J. R. Carapetis, A. C. Steer, E. K. Mulholland, and M. Weber, "The global burden of group a streptococcal diseases," Lancet Infect Dis, vol. 5, no. 11, pp. 685-94, 2005. Carapetis, Jonathan R Steer, Andrew C Mulholland, E Kim Weber, Martin eng Research Support, Non-U.S. Gov't Review Lancet Infect Dis. 2005 Nov;5(11):685-94. doi: 10.1016/S14733099(05)70267-X.

[3] B. Remenyi, N. Wilson, A. Steer, B. Ferreira, J. Kado, K. Kumar, J. Lawrenson, G. Maguire, E. Marijon, M. Mirabel, A. O. Mocumbi, C. Mota, J. Paar, A. Saxena, J. Scheel, J. Stirling, S. Viali, V. I. Balekundri, G. Wheaton, L. Zuhlke, and J. Carapetis, "World heart federation criteria for echocardiographic diagnosis of rheumatic heart disease-an evidence-based guideline," Nat Rev Cardiol, vol. 9, no. 5, pp. 297-309, 2012. Remenyi, Bo Wilson, Nigel Steer, Andrew Ferreira, Beatriz Kado, Joseph Kumar, Krishna Lawrenson, John Maguire, Graeme Marijon, Eloi Mirabel, Mariana Mocumbi, Ana Olga Mota, Cleonice Paar, John Saxena, Anita Scheel, Janet Stirling, John Viali, Satupaitea Balekundri, Vijayalakshmi I Wheaton, Gavin Zuhlke, Liesl Carapetis, Jonathan eng R24 TW007988/TW/FIC NIH HHS/ Research Support, N.I.H., Extramural Research Support, Non-U.S. Gov't Review England Nat Rev Cardiol. 2012 Feb 28;9(5):297-309. doi: 10.1038/nrcardio.2012.7.

[4] A. C. Steer, J. Kado, N. Wilson, T. Tuiketei, M. Batzloff, L. Waqatakirewa, E. K. Mulholland, and J. R. Carapetis, "High prevalence of rheumatic heart disease by clinical and echocardiographic screening among children in fiji," J Heart Valve Dis, vol. 18, no. 3, pp. 327-335, 2009. 
[5] J. R. Carapetis, "Rheumatic heart disease in developing countries," N Engl J Med, vol. 357, no. 5, pp. 439-41, 2007. Carapetis, Jonathan R eng Comment N Engl J Med. 2007 Aug 2;357(5):439-41. doi: 10.1056/NEJMp078039.

[6] M. Saad Sultan, N. Martins, E. Costa, D. Veiga, M. J. Ferreira, S. Mattos, and M. Tavares Coimbra, "Tracking large anterior mitral leaflet displacements by incorporating optical flow in an active contours framework," Conf Proc IEEE Eng Med Biol Soc, vol. 2017, pp. 3244-3247, 2017. Saad Sultan, Malik Martins, Nelson Costa, Eva Veiga, Diana Ferreira, Manuel Joao Mattos, Sandra Tavares Coimbra, Miguel eng Conf Proc IEEE Eng Med Biol Soc. 2017 Jul;2017:3244-3247. doi: 10.1109/EMBC.2017.8037548.

[7] R. H. Webb, N. Culliford-Semmens, K. Sidhu, and N. J. Wilson, "Normal echocardiographic mitral and aortic valve thickness in children," Heart Asia, vol. 9, no. 1, pp. 7075, 2017. Webb, Rachel H Culliford-Semmens, Nicola Sidhu, Karishma Wilson, Nigel J eng England Heart Asia. 2017 Mar 21;9(1):70-75. doi: 10.1136/heartasia-2016-010872. eCollection 2017.

[8] I. Szczygielska, E. Hernik, B. Kolodziejczyk, A. Gazda, M. Maslinska, and P. Gietka, "Rheumatic fever - new diagnostic criteria," Reumatologia, vol. 56, no. 1, pp. 37-41, 2018. Szczygielska, Izabela Hernik, Elzbieta Kolodziejczyk, Beata Gazda, Agnieszka Maslinska, Maria Gietka, Piotr eng Review Poland Reumatologia. 2018;56(1):37-41. doi: 10.5114/reum.2018.74748. Epub 2018 Feb 28.

[9] A. Beaton, T. Aliku, E. Okello, S. Lubega, R. McCarter, P. Lwabi, and C. Sable, "The utility of handheld echocardiography for early diagnosis of rheumatic heart disease," $J$ Am Soc Echocardiogr, vol. 27, no. 1, pp. 42-9, 2014. Beaton, Andrea Aliku, Twalib Okello, Emmy Lubega, Sulaiman McCarter, Robert Lwabi, Peter Sable, Craig eng UL1TR000075/TR/NCATS NIH HHS/ Research Support, N.I.H., Extramural J Am Soc Echocardiogr. 2014 Jan;27(1):42-9. doi: 10.1016/j.echo.2013.09.013. Epub 2013 Oct 30.

[10] J. A. Aboulhosn, R. J. Oudiz, A. S. Dave, A. Ardehali, and D. J. Ross, "Successful tricuspid valve replacement in a patient with severe pulmonary arterial hypertension and preserved right ventricular systolic function," Case Rep Med, vol. 2009, p. 108295, 2009. Aboulhosn, Jamil A Oudiz, Ronald J Dave, Amish S Ardehali, Abbas Ross, David J eng Case Rep Med. 2009;2009:108295. doi: 10.1155/2009/108295. Epub 2009 Jun 16.

[11] W. N. McDicken, G. R. Sutherland, C. M. Moran, and L. N. Gordon, "Colour doppler velocity imaging of the myocardium," Ultrasound Med Biol, vol. 18, no. 6-7, pp. 651-4, 1992. McDicken, W N Sutherland, G R Moran, C M Gordon, L N eng England Ultrasound Med Biol. 1992;18(6-7):651-4.

[12] R. M. Lang, L. P. Badano, W. Tsang, D. H. Adams, E. Agricola, T. Buck, F. F. Faletra, A. Franke, J. Hung, L. P. de Isla, O. Kamp, J. D. Kasprzak, P. Lancellotti, T. H. Marwick, 
M. L. McCulloch, M. J. Monaghan, P. Nihoyannopoulos, N. G. Pandian, P. A. Pellikka, M. Pepi, D. A. Roberson, S. K. Shernan, G. S. Shirali, L. Sugeng, F. J. Ten Cate, M. A. Vannan, J. L. Zamorano, and W. A. Zoghbi, "Eae/ase recommendations for image acquisition and display using three-dimensional echocardiography," European Heart Journal Cardiovascular Imaging, vol. 13, no. 1, pp. 1-46, 2012. 10.1093/ehjci/jer316.

[13] A. Beaton, J. C. Lu, T. Aliku, P. Dean, L. Gaur, J. Weinberg, J. Godown, P. Lwabi, G. Mirembe, E. Okello, A. Reese, A. Shrestha-Astudillo, T. Bradley-Hewitt, J. Scheel, C. Webb, R. McCarter, G. Ensing, and C. Sable, "The utility of handheld echocardiography for early rheumatic heart disease diagnosis: a field study," Eur Heart $J$ Cardiovasc Imaging, vol. 16, no. 5, pp. 475-82, 2015. Beaton, Andrea Lu, Jimmy C Aliku, Twalib Dean, Peter Gaur, Lasya Weinberg, Jacqueline Godown, Justin Lwabi, Peter Mirembe, Grace Okello, Emmy Reese, Allison Shrestha-Astudillo, Ashley BradleyHewitt, Tyler Scheel, Janet Webb, Catherine McCarter, Robert Ensing, Greg Sable, Craig eng KL2TR000076/TR/NCATS NIH HHS/ UL1TR000075/TR/NCATS NIH HHS/ Research Support, N.I.H., Extramural Research Support, Non-U.S. Gov't England Eur Heart J Cardiovasc Imaging. 2015 May;16(5):475-82. doi: 10.1093/ehjci/jeu296. Epub 2015 Jan 5.

[14] N. Subramanian, D. Padfield, S. Thiruvenkadam, A. Narasimhamurthy, and S. Frigstad, "Automated interventricular septum thickness measurement from b-mode echocardiograms," Med Image Comput Comput Assist Interv, vol. 13, no. Pt 1, pp. 510-7, 2010. Subramanian, Navneeth Padfield, Dirk Thiruvenkadam, Sheshadri Narasimhamurthy, Anand Frigstad, Sigmund eng Germany Med Image Comput Comput Assist Interv. 2010;13(Pt 1):510-7.

[15] N. M. Dhutia, M. Zolgharni, M. Mielewczik, M. Negoita, S. Sacchi, K. Manoharan, D. P. Francis, and G. D. Cole, "Open-source, vendor-independent, automated multibeat tissue doppler echocardiography analysis," Int J Cardiovasc Imaging, vol. 33, no. 8, pp. 1135-1148, 2017. Dhutia, Niti M Zolgharni, Massoud Mielewczik, Michael Negoita, Madalina Sacchi, Stefania Manoharan, Karikaran Francis, Darrel P Cole, Graham D eng 281524/European Research Council/International FS/10/38/28268/British Heart Foundation/United Kingdom FS/12/12/29294/British Heart Foundation/United Kingdom Comparative Study Evaluation Studies Int J Cardiovasc Imaging. 2017 Aug;33(8):1135-1148. doi: 10.1007/s10554-017-1092-4. Epub 2017 Feb 20.

[16] M. S. Sultan, N. Martins, E. Costa, D. Veiga, M. J. Ferreira, S. Mattos, and M. T. Coimbra, "Real-time anterior mitral leaflet tracking using morphological operators and active contours," Proceedings of the 10th International Joint Conference on Biomedical Engineering Systems and Technologies, Vol 2: Bioimaging, pp. 39-46, 2017. Bi6gn Times Cited:0 Cited References Count:20. 
[17] A. Ahmadi, E. Mitchell, C. Richter, F. Destelle, M. Gowing, N. E. O'Connor, and K. Moran, "Toward automatic activity classification and movement assessment during a sports training session," IEEE Internet of Things Journal, vol. 2, no. 1, pp. 23-32, 2015.

[18] D. Roetenberg, H. Luinge, and P. Slycke, "Xsens mvn: full 6dof human motion tracking using miniature inertial sensors," Xsens Motion Technologies BV, Jan. 2009.

[19] Z. Chen, J. Xue, C. Wu, L. Qin, L. Liu, and X. Cheng, "Classification of vessel motion pattern in inland waterways based on automatic identification system," Ocean Engineering, vol. 161, pp. 69-76, 2018.

[20] E. Amaldi and V. Kann, "On the approximability of minimizing nonzero variables or unsatisfied relations in linear systems," Theoretical Computer Science, vol. 209, no. 1, pp. 237260, 1998.

[21] E. Marijon, M. Mirabel, D. S. Celermajer, and X. Jouven, "Rheumatic heart disease," The Lancet, vol. 379, no. 9819, pp. 953-964, 2012. Marijon, Eloi Mirabel, Mariana Celermajer, David S Jouven, Xavier eng Review England Lancet. 2012 Mar 10;379(9819):953-64. doi: 10.1016/S0140-6736(11)61171-9.

[22] T. Y. Zhang and C. Y. Suen, "A fast parallel algorithm for thinning digital patterns," Commun. ACM, vol. 27, no. 3, pp. 236-239, 1984.

[23] Y. Y. Zhang and P. S. P. Wang, "A modified parallel thinning algorithm," in [1988 Proceedings] 9th International Conference on Pattern Recognition, pp. 1023-1025 vol.2, 1988.

[24] K. Saeed, M. Rybnik, and M. Tabedzki, Implementation and Advanced Results on the Noninterrupted Skeletonization Algorithm, pp. 601-609. Berlin, Heidelberg: Springer Berlin Heidelberg, 2001.

[25] K. Saeed, M. Tabędzki, M. Rybnik, and M. Adamski, "K3m: A universal algorithm for image skeletonization and a review of thinning techniques," 2010.

[26] $\mathrm{H}$. Hsieh and $\mathrm{H}$. Andrews, "Cubic splines for image interpolation and digital filtering," IEEE Transactions on Acoustics, Speech, and Signal Processing, vol. 26, no. 6, pp. 508-517, 1978.

[27] E. Catmull and R. Rom, A class of local interpolating splines, pp. 317-326. Elsevier, 1974.

[28] C. DeBoor, A practical guide to splines. Springer, 1978.

[29] J. G. Herriot and C. H. Reinsch, "Procedures for natural spline interpolation," Communications of the Acm, vol. 16, no. 12, pp. 763-768, 1973. R4559 Times Cited:11 Cited References Count:5. 
[30] C. A. R. Hoare, "Quicksort," The Computer Journal, vol. 5, no. 1, pp. 10-16, 1962. 10.1093/comjnl/5.1.10.

[31] S. SUZUKI and K. ABE, "Topological structural analysis of digitized binary images by border following," Computer vision, graphics, and image processing, vol. 30, no. 1, pp. 32-46, 1985.

[32] A. Kalangos, M. Beghetti, D. Vala, E. Jaeggi, G. Kaya, V. Karpuz, N. Murith, and B. Faidutti, "Anterior mitral leaflet prolapse as a primary cause of pure rheumatic mitral insufficiency," The Annals of Thoracic Surgery, vol. 69, no. 3, pp. 755 - 761, 2000.

[33] E. Wold, T. Blum, D. Keislar, and J. Wheaten, "Content-based classification, search, and retrieval of audio," IEEE MultiMedia, vol. 3, no. 3, pp. 27-36, 1996.

[34] G. K. Wallace, "The jpeg still picture compression standard," Commun. ACM, vol. 34, no. 4, pp. 30-44, 1991.

[35] M. Frigo and S. G. Johnson, "The design and implementation of fftw3," Proceedings of the IEEE, vol. 93, no. 2, pp. 216-231, 2005.

[36] J. Cooley and J. Tukey, "An algorithm for the machine computation of the complex fourier series, in mathematics of computation. 9: 297-301," 1965.

[37] P. Duhamel and M. Vetterli, "Fast fourier transforms: a tutorial review and a state of the art," Signal processing, vol. 19, no. 4, pp. 259-299, 1990.

[38] P. Rodriguez, "A radix-2 fft algorithm for modern single instruction multiple data (simd) architectures," in IEEE INTERNATIONAL CONFERENCE ON ACOUSTICS SPEECH AND SIGNAL PROCESSING, vol. 3, pp. III-3220, IEEE; 1999, 2002.

[39] F. Franchetti, M. Puschel, Y. Voronenko, S. Chellappa, and J. M. Moura, "Discrete fourier transform on multicore," IEEE Signal Processing Magazine, vol. 26, no. 6, 2009.

[40] G. Strang and T. Nguyen, Wavelets and filter banks. SIAM, 1996.

[41] J. R. Quinlan, C4. 5: programs for machine learning. Elsevier, 2014.

[42] R. Pandya and J. Pandya, "C5. 0 algorithm to improved decision tree with feature selection and reduced error pruning," International Journal of Computer Applications, vol. 117, no. 16, pp. 18-21, 2015.

[43] D. Steinberg and P. Colla, "Cart: classification and regression trees," The top ten algorithms in data mining, vol. 9, p. 179, 2009.

[44] Y. Freund, R. Schapire, and N. Abe, "A short introduction to boosting," Journal-Japanese Society For Artificial Intelligence, vol. 14, no. 771-780, p. 1612, 1999. 
[45] N. Littlestone, "Learning quickly when irrelevant attributes abound: A new linear-threshold algorithm," Machine Learning, vol. 2, pp. 285-318, Apr 1988.

[46] E. Osuna, R. Freund, and F. Girosit, "Training support vector machines: an application to face detection," in Proceedings of IEEE Computer Society Conference on Computer Vision and Pattern Recognition, pp. 130-136, June 1997.

[47] T. S. Furey, N. Cristianini, N. Duffy, D. W. Bednarski, M. Schummer, and D. Haussler, "Support vector machine classification and validation of cancer tissue samples using microarray expression data," Bioinformatics, vol. 16, no. 10, pp. 906-914, 2000.

[48] S. Tong and D. Koller, "Support vector machine active learning with applications to text classification,” Journal of machine learning research, vol. 2, no. Nov, pp. 45-66, 2001.

[49] A. Statnikov, D. Hardin, I. Guyon, and C. Aliferis, "A gentle introduction to support vector machines in biomedicine," AMIA Annual Symposium Proceedings, 082009.

[50] J. Mercer, "Xvi. functions of positive and negative type, and their connection the theory of integral equations," Philosophical Transactions of the Royal Society of London A: Mathematical, Physical and Engineering Sciences, vol. 209, no. 441-458, pp. 415-446, 1909.

[51] L. Breiman, "Random forests," Machine learning, vol. 45, no. 1, pp. 5-32, 2001.

[52] T. G. Dietterich, "Ensemble methods in machine learning," in International workshop on multiple classifier systems, pp. 1-15, Springer, 2000.

[53] A. Blum and R. L. Rivest, "Training a 3-node neural network is np-complete," in Advances in Neural Information Processing Systems 1 (D. S. Touretzky, ed.), pp. 494-501, MorganKaufmann, 1989.

[54] H. Laurent and R. L. Rivest, "Constructing optimal binary decision trees is np-complete," Information processing letters, vol. 5, no. 1, pp. 15-17, 1976.

[55] L. Breiman, "Bagging predictors," Machine Learning, vol. 24, pp. 123-140, Aug 1996.

[56] T. Giorgino, "Computing and visualizing dynamic time warping alignments in $r$ : The dtw package," Journal of Statistical Software, Articles, vol. 31, no. 7, pp. 1-24, 2009.

[57] P. Senin, "Dynamic time warping algorithm review," Information and Computer Science Department University of Hawaii at Manoa Honolulu, USA, vol. 855, pp. 1-23, 2008.

[58] D. P. Huttenlocher, G. A. Klanderman, and W. J. Rucklidge, "Comparing images using the hausdorff distance," IEEE Transactions on Pattern Analysis and Machine Intelligence, vol. 15, pp. 850-863, Sept 1993. 
[59] P. Rotter, A. M. Skulimowski, C. Kotropoulos, and I. Pitas, "Fast shape matching using the hausdorff distance," Mirage: Computer Vision/Computer Graphics Collaboration Techniques and Applications, INRIA Rocquencourt, France (March 2005), 2005.

[60] O. Jesorsky, K. J. Kirchberg, and R. W. Frischholz, "Robust face detection using the hausdorff distance," in Audio- and Video-Based Biometric Person Authentication (J. Bigun and F. Smeraldi, eds.), (Berlin, Heidelberg), pp. 90-95, Springer Berlin Heidelberg, 2001.

[61] C. Elkan, "The foundations of cost-sensitive learning," in International joint conference on artificial intelligence, vol. 17, pp. 973-978, Lawrence Erlbaum Associates Ltd, 2001.

[62] M. S. Sultan, N. C. Martins, E. Costa, D. Veiga, M. J. Ferreira, S. Mattos, and M. T. Coimbra, "Virtual m-mode for echocardiography: A new approach for the segmentation of the anterior mitral leaflet," IEEE Journal of Biomedical and Health Informatics, pp. 1-1, 2018. 